\title{
THESIS
}

\section{DESK-TOP SIZE HIGH REPETITION RATE 46.9 NM CAPILLARY DISCHARGE LASER AS PHOTOIONIZATION SOURCE FOR PHOTOCHEMISTRY APPLICATIONS}

\author{
Submitted by \\ Scott Heinbuch \\ Department of Electrical and Computer Engineering
}

In partial fulfillment of the requirements

For the Degree of Master of Science

Colorado State University

Fort Collins, Colorado

Spring 2006 
COLORADO STATE UNIVERSITY

FEBRUARY 13, 2006

WE HEREBY RECOMMEND THAT THE THESIS PREPARED UNDER OUR SUPERVISION BY SCOTT HEINBUCH ENTITLED DESK-TOP SIZE HIGH REPETITION RATE 46.9 NM CAPILLARY DISCHARGE LASER AS PHOTOIONIZATION SOURCE FOR PHOTOCHEMISTRY APPLICATIONS BE ACCEPTED AS FULFILLING IN PART REQUIREMENTS FOR THE DEGREE OF MASTER OF SCIENCE.
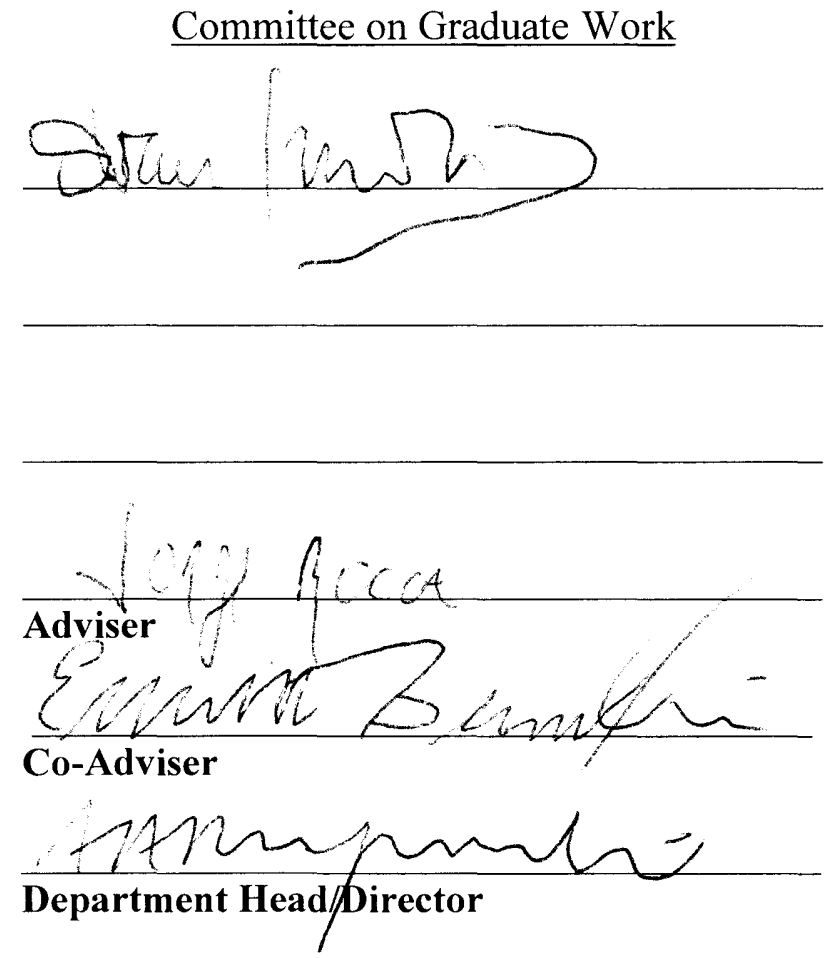

ii 


\section{ABSTRACT OF THESIS \\ DESK-TOP SIZE HIGH REPETITION RATE 46.9 NM CAPILLARY DISCHARGE LASER AS PHOTOIONIZATION SOURCE FOR PHOTOCHEMISTRY APPLICATIONS}

A portable high repetition rate desktop-size capillary discharge laser emitting at a wavelength of $46.9 \mathrm{~nm}$ (26.5 $\mathrm{eV}$ photon energy) was demonstrated and used as a photoionization source in nanocluster mass spectroscopy. The high photon energy allows the single-photon ionization of nanoclusters and other molecules, which, due to their high ionization potential, would otherwise require undesirable multi-photon ionization. This Ne-like Ar capillary discharge laser occupies a table area of approximately $0.4 \times 0.4 \mathrm{~m}^{2}$, smaller than that occupied by many widely used ultraviolet gas lasers. The laser's power supplies and gas handling system are designed to fit into small racks that can be placed underneath a standard optical table. The main spark-gap is electrically triggered to allow synchronization of the laser pulses with those of other lasers in photochemistry applications.

Experiments were performed to characterize the laser output energy, average power and timing jitter. Tests were conducted to determine the capillary lifetime. Laser pulses with energy $\sim 13 \mu \mathrm{J}$ were generated at $12 \mathrm{~Hz}$ repetition rate by single pass amplification in a $21 \mathrm{~cm}$ long Ne-like Ar capillary discharge plasma column. The standard deviation of the jitter was found to be $5 \mathrm{~ns}$. Capillary lifetime tests at $12 \mathrm{~Hz}$ repetition rate determined that the laser output energy decays by a factor of two after about $210^{4}-310^{4}$ shots.

The laser was installed in a photochemistry laboratory where it is operated for many hours on a daily basis. The laser was successfully used as a single photon 
photoionization source for the study of hydrogen bonded nanoclusters and other small molecules using time of flight mass spectroscopy. The first mass spectra of water nanoclusters and other small molecules using this source have been obtained.

Scott Heinbuch Electrical and Computer Engineering Department Colorado State University Fort Collins, CO 80523 Spring 2006 


\section{CHAPTER 1}

\section{INTRODUCTION}

\section{OVERVIEW OF TABLE-TOP SOFT X-RAY LASERS}

\subsection{Introduction to soft $x$-ray lasers}

Since the first demonstration of soft x-ray lasing using the collisional excitation mechanism in neon-like selenium [1.1] significant efforts have been devoted to reduce the size of soft x-ray lasers from laboratory size [1.2] to table-top [1.3-1.7]. There exists numerous potential applications spanning many different fields of study that can benefit from the use of compact short wavelength lasers. In this chapter, the need for compact coherent short wavelength sources is established through the discussion of applications that motivate their development. The second chapter discusses the development and demonstration of a $46.9 \mathrm{~nm}$ wavelength desk-top size laser driven by a capillary discharge, and the following chapter demonstrates its use as a single photon ionization source in the time of flight mass spectroscopy study of nanoclusters.

A definition of the extreme ultraviolet (EUV)/soft $\mathrm{x}$-ray region of the spectrum is relevant to the upcoming work. The EUV region is loosely defined as photon energies of $\sim 25 \mathrm{eV}$ to $\sim 250 \mathrm{eV}$, with corresponding wavelengths extending from $\sim 50 \mathrm{~nm}$ to $5 \mathrm{~nm}$ [1.8]. The soft $\mathrm{x}$-ray region is usually taken as extending from about $250 \mathrm{eV}$ to several $\mathrm{keV}$ as seen in figure 1.1 although it should be noted that the "x-ray laser" community often defines lasers with wavelengths shorter that about $50 \mathrm{~nm}$ as soft $\mathrm{x}$-ray lasers. Due to the lack of a precise definition of the boundaries of these regions, this thesis will use the terms soft x-ray and EUV interchangeably. Also, note the region between the two 
vertical dashed lines in figure 1.1. It contains the ionization energies for the nanoclusters that are a major topic of this thesis reported in chapter 3.

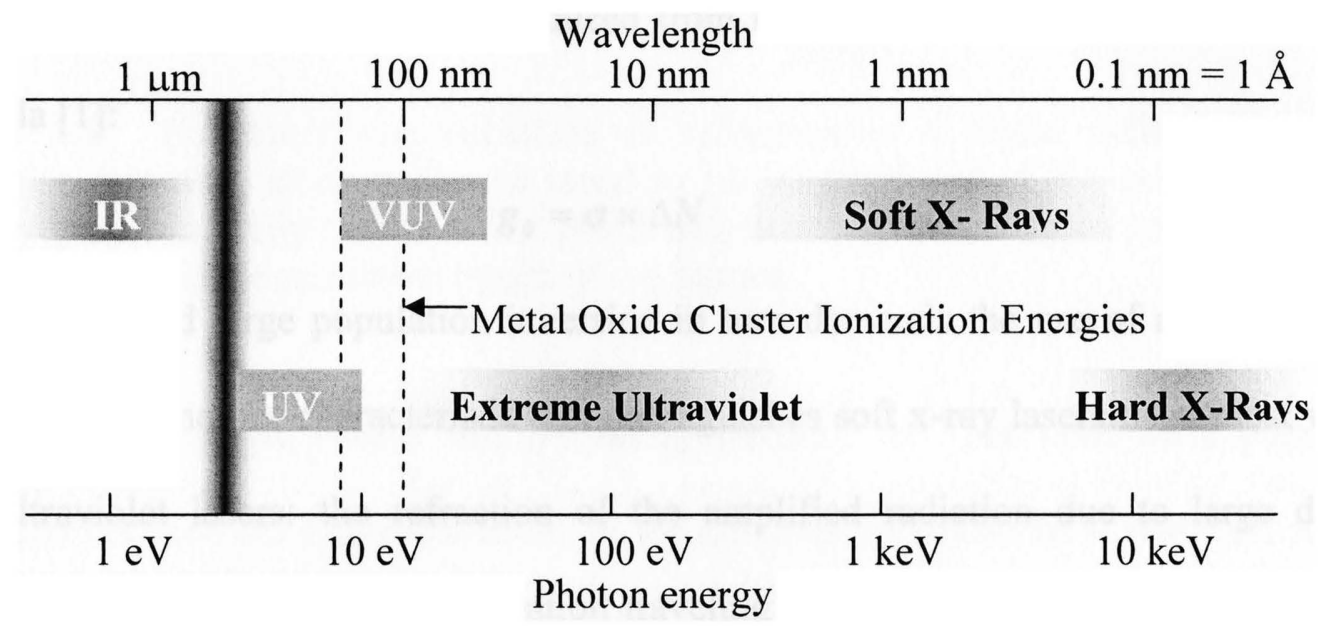

Figure 1.1: Electromagnetic spectrum from the infrared to the $\mathrm{x}$-ray regions. At short wavelengths we find the vacuum ultraviolet (VUV), extreme ultraviolet (EUV), soft xrays (SXR), and hard x-rays. The region in between the vertical dashed lines contains typical ionization energies for a wide variety of nanocluster systems. (Figure modified from reference [8]).

Some general considerations need to be taken into account when discussing short wavelength lasers. In visible and UV lasers it is possible to generate large amplification by passing the light through the amplifying medium several times with the use of a cavity created by highly reflective mirrors. In the case of soft x-ray lasers the duration of the gain is very short (typically between 10 ps to $1 \mathrm{~ns}$ ) and the reflectivity of materials at normal incidence is extremely low, making a conventional mirror cavity virtually impossible. The result is a single pass amplification laser that operates based on the amplification of spontaneous emission in a gain medium that is a dense plasma. Occasionally, use of a double pass configuration is accomplished using a mirror at one end of the amplifying medium creating a half cavity. The need of achieving large 
amplification in a single pass through the gain medium requires a large gain coefficient $\mathrm{g}_{0}$. This in turn results in the requirement for a large population inversion, $\Delta \mathrm{N}$, due to the relatively small value of the stimulated emission cross section, $\sigma$, associated with short wavelength transitions, as can be deduced from the relation between these variables in formula [1]:

$$
g_{0}=\sigma \times \Delta N
$$

The required large population inversion in turn demands the use of a dense plasma that results in another characteristic that distinguishes soft $\mathrm{x}$-ray lasers from most visible and ultraviolet lasers: the refraction of the amplified radiation due to large density gradients in the gain medium. Radiation traveling along a plasma tends to be refracted out of the central region at an angle given by formula [2]:

$$
\theta=\sqrt{\frac{n_{e}}{n_{c}}}
$$

where $n_{e}$ is the maximum electron density along the axis and $n_{c}$ is the critical density at the wavelength of light given by $n_{c}=10^{21} \times \lambda^{-2} \mathrm{~cm}^{-3}$, where $\lambda$ is in microns. This is illustrated in figure 1.2. At our wavelength of interest, $46.9 \mathrm{~nm}$, the critical density is 5 $10^{23} \mathrm{~cm}^{-3}$ and a maximum electron density of $\sim 10^{19}$ is assumed from theoretical calculations discussed later in this chapter, giving a refraction angle of $\sim 4.5 \mathrm{mrad}$. This value is in good agreement with the experimental value that is reported in chapter 2 . With the presence of strong refraction, only radiation that propagates near the axis experiences substantial gain and contributes to the output of the laser. Aside from single pass amplification and the operation in transitions of multiply ionized atoms where the energy level separation is greater than $25 \mathrm{eV}$, soft $\mathrm{x}$-ray lasers are based on many of the same 


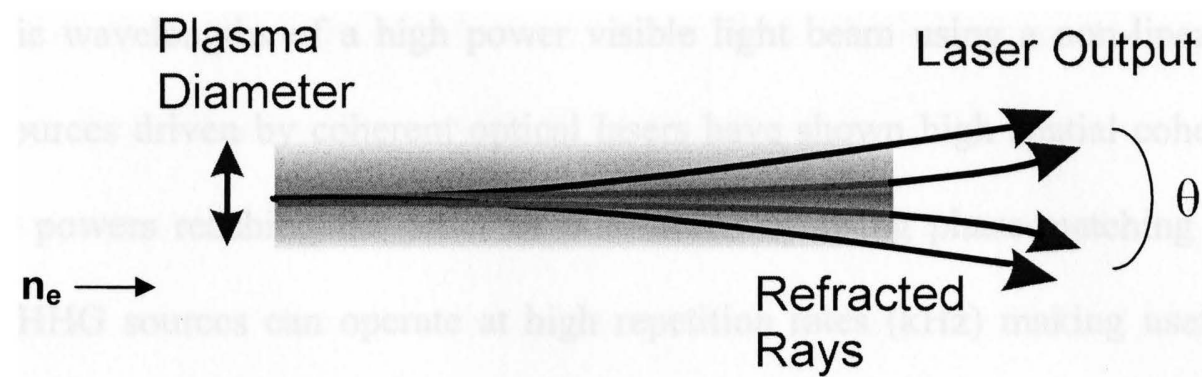

Figure 1.2: Schematic representation of refraction in a dense cylindrical plasma. Radiation traveling along a plasma tends to be refracted out of the central region by density gradients. Only radiation that propagates near the center region contributes to the laser output. $\mathrm{L}_{\mathrm{r}}$ is the refraction length of the plasma.

principals that typical visible and UV lasers are built on. The need for a population inversion between the upper and lower levels of the lasing transition is required, and can be obtained in a similar way as in established visible gas laser schemes. Electron collision $[1.1-1.4,1.6-1.8]$ and collisional recombination $[1.9,1.36-1.37]$ excitation of the laser upper level are normally used as population inversion mechanisms. In soft x-ray lasers, the ionization and upper laser level excitation are most frequently produced by electron impact, although the use of a process known as optical field ionization in which the atoms are ionized by the depression of the Coulomb barrier in an intense optical field has been demonstrated as well $[1.5,1.31-1.35]$. The next section will discuss the generation of soft x-ray light and some of the excitation methods that have been demonstrated to date.

\subsection{Generation of coherent soft $x$-ray light}

The degree of spatial coherence of radiation plays a critical role in many important applications as illustrated in the upcoming sections of this chapter. Current approaches for the generation of high brightness coherent radiation in the soft $\mathrm{x}$-ray spectral region include high-order harmonic generation (HHG) of optical lasers [1.18-1.20], and soft xray lasers [1.21-1.24]. The technique of HHG generates radiation at multiple odd 
harmonic wavelengths of a high power visible light beam using a non-linear medium. HHG sources driven by coherent optical lasers have shown high spatial coherence with average powers reaching the order of microwatts by using phase-matching techniques [1.16]. HHG sources can operate at high repetition rates $(\mathrm{kHz})$ making useful average powers attainable. However, they have a low energy per pulse, typically in the $\mathrm{nJ}$ to $\mu \mathrm{J}$ range. In contrast, $\mathrm{x}$-ray lasers have the potential to generate pulses with energy in the $\mathrm{mJ}$ level. Before discussing methods of generating short wavelength radiation, the difference between coherent versus incoherent light is briefly described.

Coherence is a property of waves that measures their ability to interfere. Ordinary light from the Sun, light bulbs, etc consists mainly of random phase light waves, that unless "filtered" is incoherent. Coherent light, normally produced by lasers is characterized by wavefronts that maintain a fixed phase relationship that gives them the natural ability to interfere. Two waves that are coherent can be combined to produce an unmoving distribution of constructive and destructive interference depending on the relative phase of the waves at their meeting point. Waves that are incoherent, when combined, produce rapidly moving areas of constructive and destructive interference and therefore do not produce a visible interference pattern. Spatial coherence is the ability of any one spatial position of the wavefront to interfere with any other spatial position and is related to the size of the light source. A point source of zero diameter emits spatially coherent light, while the light from a source of finite diameter would have lower coherence. Spatial coherence can be increased with a spatial filter consisting of a very small pinhole preceded by a condenser lens. The spatial coherence of light increases as it 
travels away from the source and becomes more like a sphere or plane wave, as described by the van Cittert-Zernike Theorem [1.8, 1.11-1.14].

A wave can also be coherent for a certain period of time, a property known as temporal coherence. If a wave is combined with a delayed copy of itself (as in a Michelson interferometer), the duration of the delay over which it produces visible interference is known as the coherence time of the wave, $\Delta t_{\mathrm{c}}$. From this, a corresponding coherence length can be calculated: $\Delta x_{c}=c \cdot \Delta t_{c}$, where $c$ is the speed of the wave. The temporal coherence of a wave is inversely proportional to the spectral bandwidth of the source. An ideally monochromatic (single frequency) wave would have an infinite coherence time and length. In practice, no wave is truly monochromatic, but in general, the coherence time can be large.

Light from common sources (such as light bulbs) is not monochromatic and has a very short coherence length $(\sim 1 \mu \mathrm{m})$, and can be considered totally temporally incoherent for most purposes. Light waves produced by a laser often have high temporal and spatial coherence, though the degree of coherence depends strongly on the properties of the specific laser. For example, a single mode helium-neon laser can produce light with coherence lengths in excess of $5 \mathrm{~m}$. The following section will discuss the methods for creating coherent $\mathrm{x}$-ray laser sources.

\subsubsection{Coherent soft $x$-ray laser generation}

In soft x-ray lasers, an optical laser pulse or an energetic electrical discharge excites a target (solid or gas), stripping its atoms of electrons to form ions and pumping energy into the ions. The first collisional x-ray laser, demonstrated by Matthews et al in an experiment conducted at Lawrence Livermore National Laboratory, was based on the 
creation of population inversion by collisional electron excitation in a Se laser produced plasma [1.1]. In that experiment $\sim 500 \mathrm{~J}$ pulses from a high power optical laser were line focused onto a $750 \AA$ Selenium (Se) foil, exploding the target creating a Ne-like Se plasma that emits in the soft x-ray region $(206.3 \AA)$. Neon-like ions in a plasma are very stable, closed-shell ions. A simplified energy level diagram for Ne-like Se is illustrated in figure 1.3 [1.25]. Excitation of the laser upper level is achieved through direct monopole excitation to the $3 p \mathrm{~J}=0$ level, and cascades from upper levels to the $3 p \mathrm{~J}=2$ levels. Radiative decay from the $3 p$ levels to ground state is forbidden. On the contrary, the $3 \mathrm{~s}$ $\mathrm{J}=1$ levels decay very quickly leading to a population inversion between the $3 \mathrm{p}$ and $3 \mathrm{~s}$ levels.

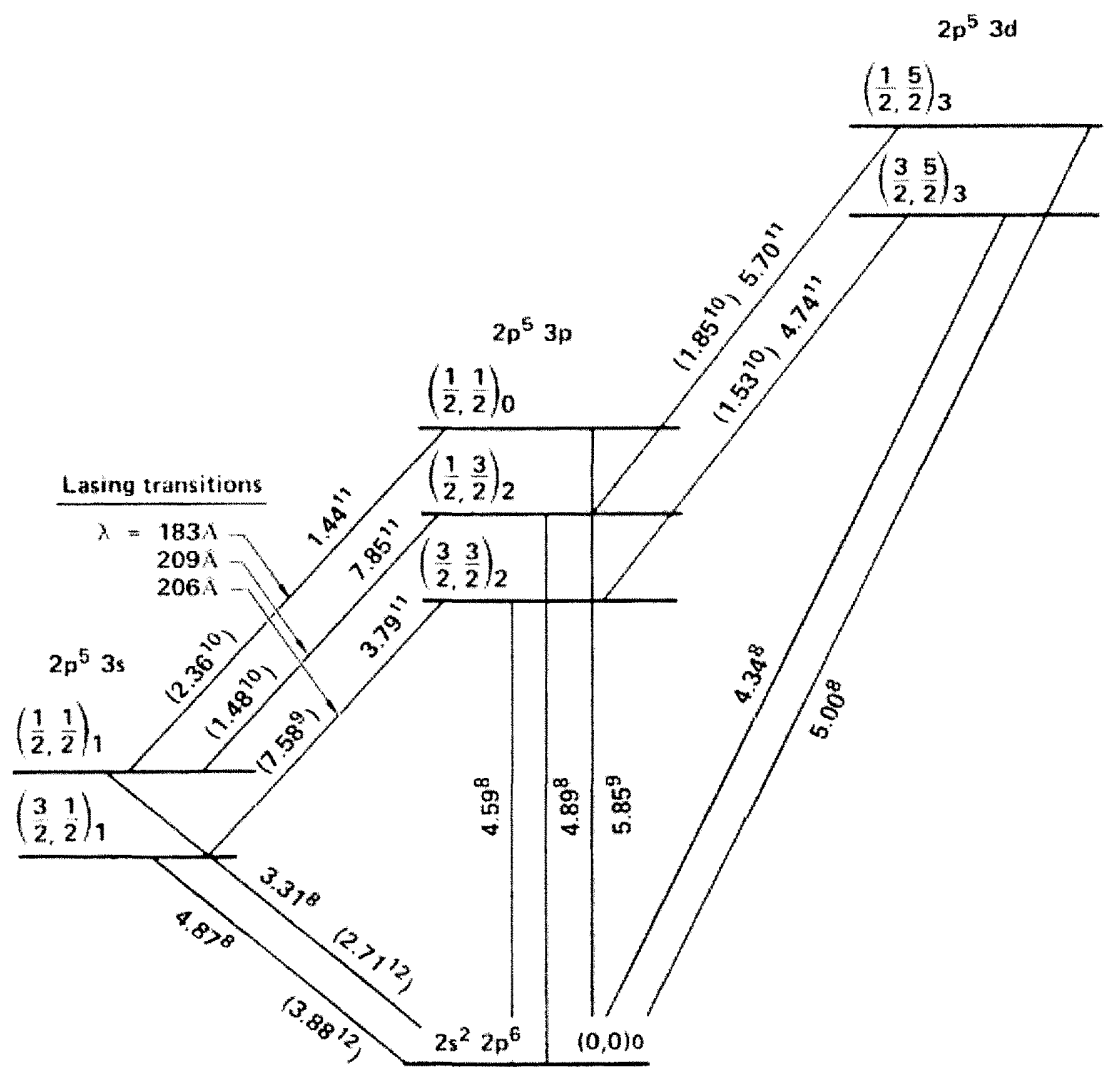

Fig. 1.3: Simplified energy level diagram for Ne-like Se. Collisional excitation rates and radiative decay rates (in parentheses) are shown for $n_{\mathrm{e}}=510^{20} \mathrm{~cm}^{-3}$ and $\mathrm{T}_{\mathrm{e}}=1 \mathrm{keV}$. (Figure is from reference 1.25 ) 
Nearly simultaneously with the first demonstration of the collisional scheme, the recombination method was established by Suckewer et al at Princeton's Plasma Physics laboratory [1.9]. To date, there have been many recombination experiments conducted, however no soft $\mathrm{x}$-ray recombination laser has been demonstrated in the saturated regime [1.36-1.37]. Since this limits their use in applications this excitation mechanism is only briefly mentioned here. The recombination laser scheme is conceptually very simple. A plasma of fully stripped ions is rapidly cooled, most commonly through the process of adiabatic expansion. The low electron temperatures created result in three-body processes dominating the recombination, preferentially filling highly excited states. The generation of an inversion between the rapidly populated upper and lower laser levels ensues if the cooling is sufficiently fast.

The demonstration of amplification using the laser collisional scheme demonstrated by Matthews et al and the recombination method by Suckewer et al are significant milestones in soft x-ray history. However, these two lasers required very large pump energies, $0.3-1 \mathrm{~kJ}$, and because of the large size and cost of the pump lasers, they were impractical. Consequently, this led to efforts that focused on new pumping schemes that reduce the pump energy required to produce soft $x$-ray radiation, ultimately leading to decreased cost and increased availability. Two approaches were followed: new laser plasma excitation schemes that require less heating energy, and the use of a fast discharge in a capillary channel to create the plasma column. The capillary discharge method will be discussed in more detail in the next section. The rest of this section will be devoted to laser plasma excitation processes. 


\subsubsection{Soft X-ray Lasers in Laser Created Plasmas: The Prepulse technique}

An important reduction of the energy necessary to excite laser-driven collisional lasers was achieved by using multiple optical laser pulses to create the plasma, commonly referred to as the prepulse technique [1.27-1.28]. This method introduces a low-intensity prepulse that precedes a main optical drive pulse by a specific time interval. The beam is focused into a line onto a target. The prepulse creates a pre-plasma that is allowed to expand over the duration of the time interval between pulses in order to 1) optimize uniformity of the plasma density at the densities required for lasing, 2) provide a larger gain region, and most importantly 3 ) reduce electron density gradients. Finally, the main heating pulse reheats the plasma, and through electron collisions, it populates the laser upper level. J. Nilsen et al demonstrated the prepulse technique in 1993 using the Nova laser at LLNL by creating a 600 ps FWHM, 6J prepulse that preceded the $1100 \mathrm{~J}$ main pulse by 7 ns [1.27]. The laser pulses were line focused onto iron, chromium and titanium targets creating laser amplification at $25.5 \mathrm{~nm}, 28.5 \mathrm{~nm}$, and $32.6 \mathrm{~nm}$ respectively. A reduction of the input power required to pump a Ne-like Germanium soft x-ray laser occurred in 1996 when E. Fill et al used $450 \mathrm{~J}$, main pulse plus full prepulse, from the Asterix IV Iodine laser at the Max-Planck-Institut für Quantenoptik [1.28]. Germanium slabs were irradiated resulting in $\mathrm{J}=0-1$ and $\mathbf{J}=2-1$ transitions at $19.6,23.2$, and $23.6 \mathrm{~nm}$.

\subsubsection{Transient heating}

Another variation based on the prepulse scheme makes use of a transient heating pulse that is short compared to the lifetime of the laser transition [1.30]. This creates a large population inversion for a short amount of time where extremely large gain can be 
achieved. P.V. Nickles et al demonstrated this in 1997 by using a long $7 \mathrm{~J}, 1.5 \mathrm{~ns}$ prepulse that interacts with a solid target of $\mathrm{Ti}$, followed by a second $0.7 \mathrm{ps}, 4 \mathrm{~J}$ pulse that rapidly heats the pre plasma and excites the laser upper level via electron collisions on a time scale shorter than the radiative and collisional relaxation time of the upper laser level. This resulted in gains of $\sim 19 \mathrm{~cm}^{-1}$ in a laser transition of $32.6 \mathrm{~nm}$. This method was later improved by J. Dunn et al when their group demonstrated small signal gain saturation on several transient-gain Ni-like ion x-ray lasers by using a high-power, chirped-pulse amplification, tabletop laser [1.4] as the drive laser. These results have been achieved at wavelengths from 139-203 $\AA$ using a total of 5-7 J energy. Gain of 62 $\mathrm{cm}^{-1}$ and GL product of 18 are determined on the $4 d \rightarrow 4 p$ transition for Ni-like Pd at 147 $\AA$ with an output energy of $12 \mu \mathrm{J}$.

\subsubsection{Grazing incidence pumping}

Different pumping configurations have been investigated to further reduce the necessary pumping energy and enable operation at higher repetition rate. Transverse excitation of a Mo target with $150 \mathrm{fs}, 300 \mathrm{~mJ}$ pulses impinging at 60 degrees from normal incidence resulted in the appearance of the 18.9 and $22.6 \mathrm{~nm}$ laser lines of Ni-like Mo [1.67]. Longitudinal pumping at $10 \mathrm{~Hz}$ produced non-saturated amplification at $18.9 \mathrm{~nm}$ in Ni-like Mo [1.68]. Recently, it has been demonstrated that in transverse excitation experiments the energy deposition efficiency of the short pulse can be significantly increased by directing it at grazing incidence [1.69-1.72]. This geometry, which inherently provides traveling wave pumping, increases the path length of the rays in the gain region of the plasma, thereby increasing the fraction of the pump energy absorbed in that region. Pumping of the $18.9 \mathrm{~nm}$ line of Ni-Mo with $150 \mathrm{~mJ}$ of total pumping energy 
from a $10 \mathrm{~Hz}$ laser, Keenan et al. reported to generate a gain-length product of $\sim 14$ [1.71], and Luther et al. used $1 \mathrm{~J}$ heating pulses to obtain in operation of the same transition in the gain-saturated regime [1.72]. Wang et al. used transient grazing incidence pumping with $1 \mathrm{~J}$ pulses to demonstrate $5 \mathrm{~Hz}$ repetition rate operation of saturated table-top lasers in Ni-like $\mathrm{Ru}, \mathrm{Pd}$ and $\mathrm{Ag}$ ions at wavelengths of 16.5, 14.7, and $13.9 \mathrm{~nm}$ respectively [1.73]. Amplification was also observed for shorter wavelength transitions of the same isolectronic sequence, with amplification in the $11.9 \mathrm{~nm}$ line of Ni-like Sn approaching gain saturation, and progressively reduced gain for wavelengths as low as $10.9 \mathrm{~nm}$ for Ni-like Te.

\subsubsection{Optical field ionization}

Another soft x-ray laser excitation scheme consists in the optical field ionization (OFI) of a moderate-density gas by a high-intensity short laser pulse to create a nonequilibrium plasma in which population inversion between excited states of specific ionic species can be obtained [1.31]. The wavelength and the polarization of the driving laser have large effects on free-electron kinetic energy distribution [1.32-1.33]. If linear polarization is used, the mean electron energy is minimized, which promotes strong recombination. In contrast, the electrons released by a circularly polarized laser field retain a kinetic energy that is equal to the quiver energy of the electric filed at the time of ionization creating a hot plasma. The resultant hot electron distribution can be suitable for pumping the upper level of the laser transition by collisional excitation [1.31]. In 1995, Lemoff et al. first demonstrated this scheme in Pd-like Xe at $41.8 \mathrm{~nm}$ [1.65]. In 1999, Sebban et al succeeded in demonstrating lasing in $\mathrm{Xe}^{8+}$ at $41.8 \mathrm{~nm}$ by focusing a $330-\mathrm{mJ}$ 35 -fs circularly polarized laser pulse into a gas cell filled with 15 torr of xenon [1.5]; 
saturated amplification was demonstrated for the first time using the collisional OFI XRL technique. More recently, the same group extended the OFI XRL technique to transitions in Ni-like ions. Strong amplification at $32.8 \mathrm{~nm}$ on the transition in $\mathrm{Kr}$ has been observed [1.34-1.35].

This section summarized methods of plasma excitation using a laser driver. The next section is dedicated to the discussion of the capillary discharge scheme which makes use of fast electrical discharges. This idea has shown significant progress in decreasing the size of soft $\mathrm{x}$-ray lasers operating in the $46.9-60.8 \mathrm{~nm}$ spectral region and is the topic of the work conducted in this thesis.

\subsection{Capillary discharge lasers}

An efficient method of creating a coherent soft x-ray source is to use an electric discharge to generate a plasma with optimum conditions for lasing. The demonstration of laser amplification in transitions of Ne-like ions in a capillary discharge plasma at Colorado State University $[1.3,1.38]$ opened the possibility to develop very compact short wavelength lasers for applications. The basic ideas and physics of a capillary discharge pumped laser are presented as in this section. We begin by introducing the processes that create soft $\mathrm{x}$-ray laser amplification in a discharge created plasma. Subsequently, we will look at the design of the early capillary discharge lasers, and how they evolved into the compact desk-top laser that is the topic of this thesis.

\subsubsection{The capillary discharge process}

Consider two electrodes at the ends of a dielectric capillary as seen in figure 1.5 , where a gas fills the capillary bore. To create a plasma a high voltage pulse is applied between the electrodes, creating a current pulse that flows between the electrodes. The resulting 
Lorentz force acting on the plasma compresses it toward the axis of the capillary. The capillary geometry forces the plasma to take on a radial symmetry; hence the partiallyionized gas medium is under radial compression. While being compressed, the plasma column becomes hotter and increasingly dense (adiabatic compression). The plasma is compressed until the particle pressure balances the magnetic pressure. At this point the plasma column reaches its minimum diameter, and the time at which it occurs may be defined as the time of the pinch. In plasma physics, this compression phenomenon is called a z-pinch and takes place when the magnetic pressure due to the interaction of a current and the magnetic field induced by the current is larger that the particle pressure. A z-pinch is a viable way to generate high-temperature and high-density plasmas, which are useful not only for soft x-ray laser sources, but are also of interest for the generation
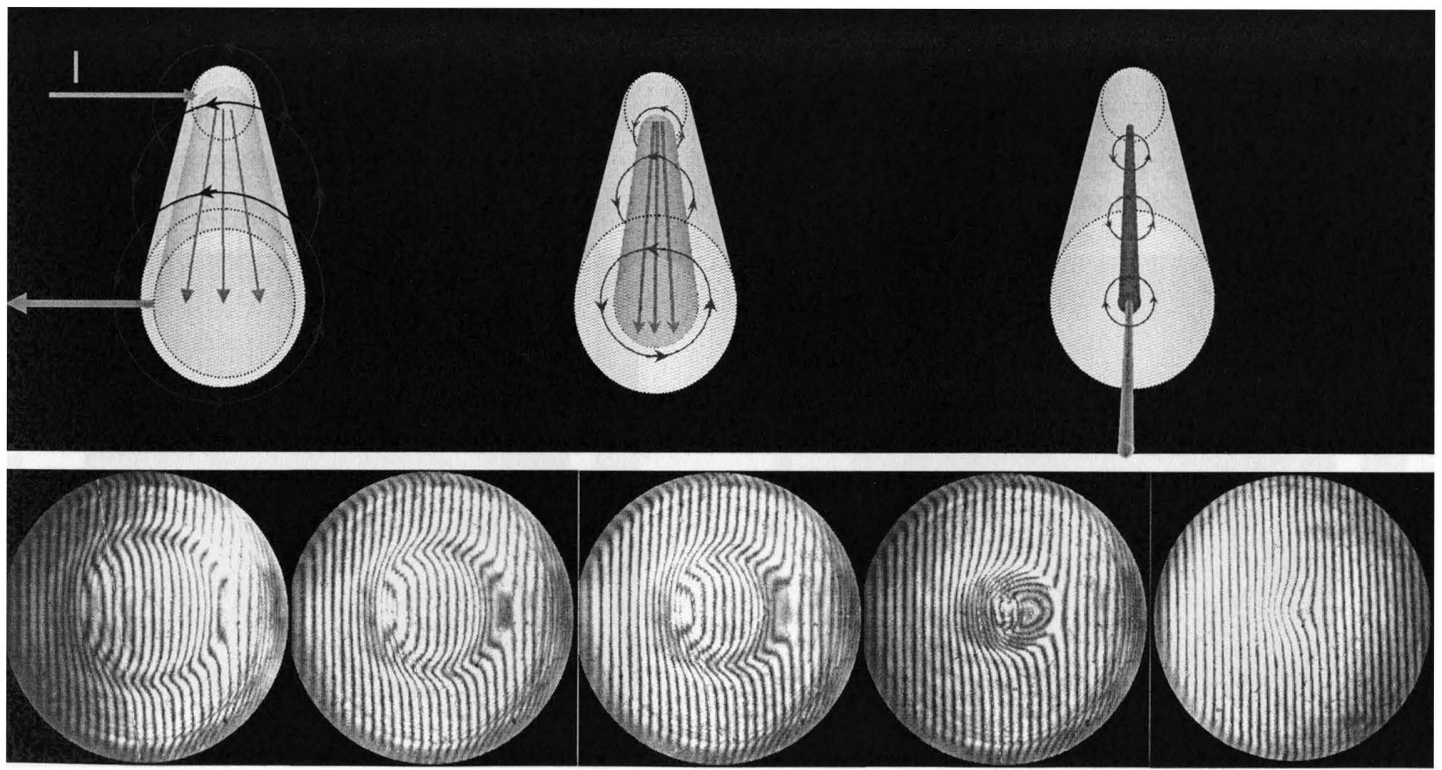

Figure 1.5: Schematic illustration of plasma compression in a fast capillary discharge. The magnetic field produced by the current generates a Lorentz force that rapidly compresses the plasma towards the axis. The sequence of end on interferograms shown in the lower part of the figure illustrate the evolution of the collapsing plasma in an Ar discharge [1.66]. The plasma column pinches on axis creating a plasma about 200-300 um in diameter where laser action takes place by electron impact excitation of multiply ionized atoms. 
of incoherent short wavelength light and even for nuclear fusion [1.39].

This phenomenon is illustrated in figure 1.6 with the results of a hydrodynamic model created by Mark Berrill at Colorado State University. The results illustrate the evolution of an Ar plasma excited by $22 \mathrm{kA}$ current pulses in a $3.2 \mathrm{~mm}$ capillary tube. These are the discharge conditions used for the development of the laser that is the topic of this thesis. Figure 1.6a illustrates the evolution of different zones of the plasma with equal mass over the rise time of the current pulse (shown in red). As the current pulse increases, the
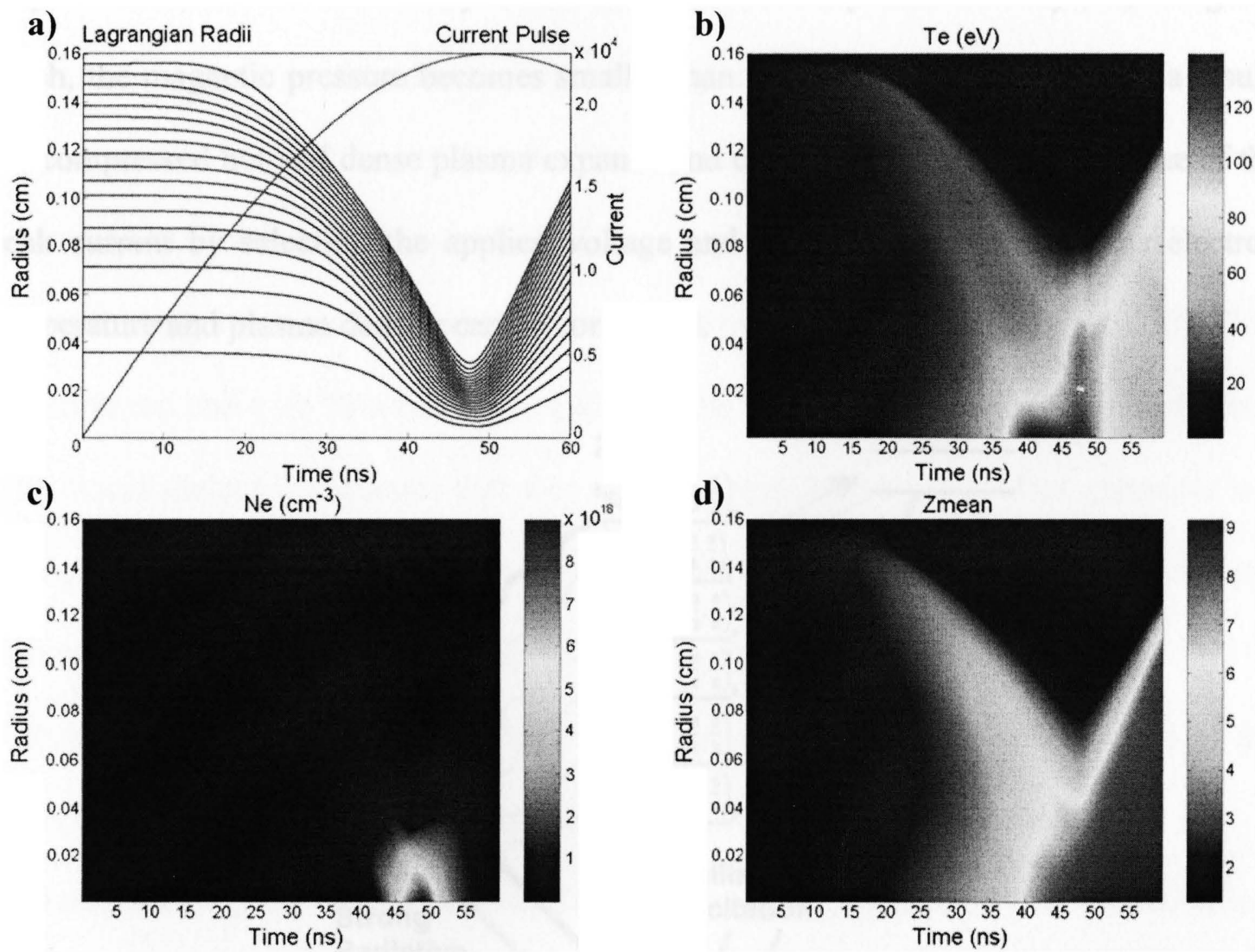

Figure 1.6: Computed Evolution of an Ar Capillary discharge plasma generated in a 3.2 $\mathrm{mm}$ diameter capillary by a fast current pulse with $22 \mathrm{kA}$ peak amplitude (current pulse in red). The initial pressure is assumed to be $700 \mathrm{mTorr}$. a) Evolution of different zones of the plasma column with equal mass. At the time of the pinch that the electron temperature reaches $\sim 100 \mathrm{eV}(\mathrm{b})$, and the electron density is increased to $\sim 810^{18} \mathrm{~cm}^{-3}$ (c) and the degree of ionization reaches $8^{+}(\mathrm{d})$, corresponding to the Ne-like Ar state. Calculations were done by Mark Berrill (CSU). 
plasma column compresses radially until it finally pinches on axis creating electron temperatures of $80-100 \mathrm{eV}(1.6 \mathrm{~b})$. At the same time the electron density reaches $\sim 810^{18}$ $\mathrm{cm}^{-3}(1.6 \mathrm{c})$. These conditions create eight times ionized Ar atoms (1.6d), corresponding to the Ne-like state of Ar, that are excited to the laser upper level by electron impact excitation, creating a large population inversion between the $3 \mathrm{p}{ }^{1} \mathrm{~S}_{0}$ laser upper level and $3 \mathrm{~s}{ }^{1} \mathrm{P}_{1}$ lower level (figure 1.7). The lower level is depopulated by strong radiative decay to the Ne-like ground state. The energy difference in the levels produces laser amplification at $46.9 \mathrm{~nm}$ shortly before the time of the pinch. Directly following the pinch, the magnetic pressure becomes smaller than the particle pressure, and as a result, the compressed hot and dense plasma expands and cools. By controlling the value of the peak current by selecting the applied voltage and the initial gas density, the electron temperature and plasma density can be controlled.

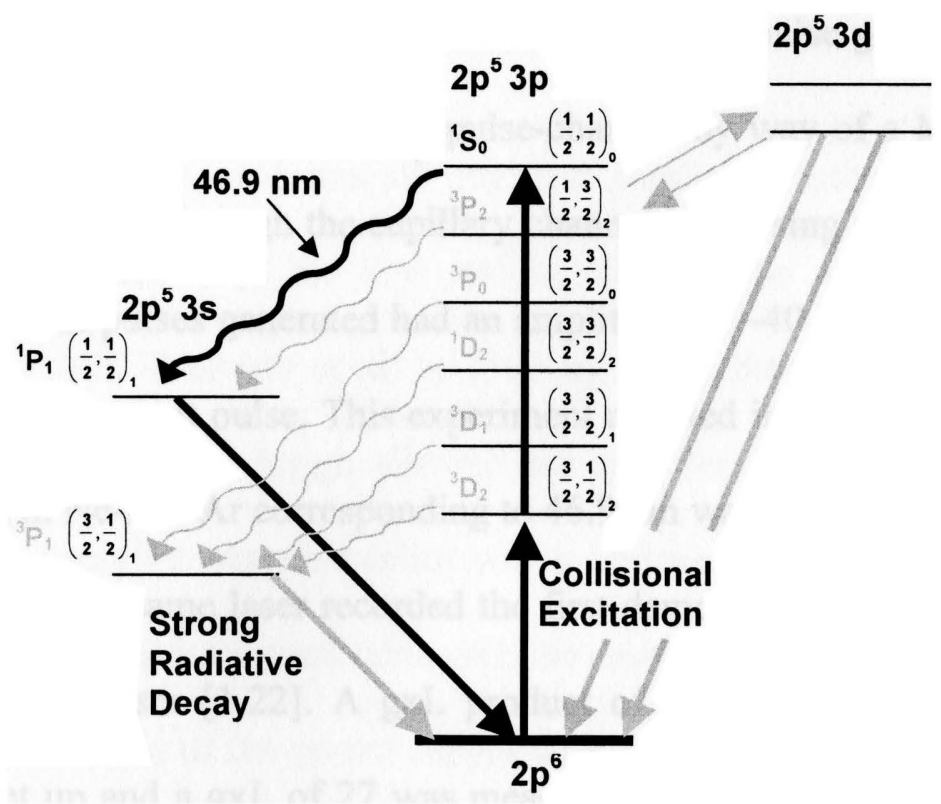

Figure 1.7: Simplified energy level diagram for Ne-like Ar. Population inversion is created by electron impact excitation from the Ne-like Ar ground state to the $3 \mathrm{p}^{1} \mathrm{~S}_{0}$ laser upper level creating a strong population inversion with respect to the $3 \mathrm{~s}{ }^{1} \mathrm{P}_{1}$ lower level, producing lasing at $46.9 \mathrm{~nm}$. Depopulation of the lower level is caused by strong radiative decay to the ground state. 


\subsubsection{Evolution in capillary discharge laser design}

Table-top size Ne-like Ar lasers operating at a wavelength of $46.9 \mathrm{~nm}$ were first developed making use of capillary discharges driven by large liquid dielectric capacitors that are pulse-charged to high voltage $(>200 \mathrm{kV})$ by multi-stage voltage amplifiers known as Marx generators (schematic in figure 1.8) [1.38, 1.40-1.41]. These lasers have been used in numerous applications, including interferometry of dense plasmas [1.42], the measurement of optical constants [1.43], materials ablation [1.44], the characterization of soft x-ray optics [1.45], nanopatterning [1.46], and imaging [1.54]. J. J. Rocca et al made the first demonstration of high soft $\mathrm{x}$-ray amplification in a discharge created plasma in 1994 in which a Ne-like Ar soft x-ray capillary discharge laser was created [1.21]. The experiment resulted in a gain length product of $\mathrm{GxL}=7.2$ was obtained using a fast discharge excitation of capillary plasmas up to $12 \mathrm{~cm}$ in length. The current pulse had a $10-90 \%$ rise time of 20 ns. The current pulse generator consisted of a $3 \mathrm{nF}$ liquid dielectric capacitor that was pulse-charged by way of a Marx generator and then quickly discharged through the capillary channel by closing a pressurized spark-gap switch. The current pulses generated had an amplitude of $\sim 40 \mathrm{kA}$ and were preceded by a $10 \mathrm{~A}$ preionizing current pulse. This experiment resulted in amplification of the $3 \mathrm{~s}^{1} \mathrm{~S}_{0^{-}}$ $3 \mathrm{p}^{1} \mathrm{P}_{1}$ transition in Ne-like Ar corresponding to $46.9 \mathrm{~nm}$ wavelength radiation.

Later, in 1996 the same laser recorded the first demonstration of gain saturation in a table-top soft $\mathrm{x}$-ray laser [1.22]. A gxL product of 14 was obtained in a single pass amplification set up and a gxL of 27 was measured with a double pass system using an Iridium mirror. The main limitation of this initial design was the large charging voltage required $(\sim 500 \mathrm{kV})$ to generate sufficient current to create the lasing plasma. This 


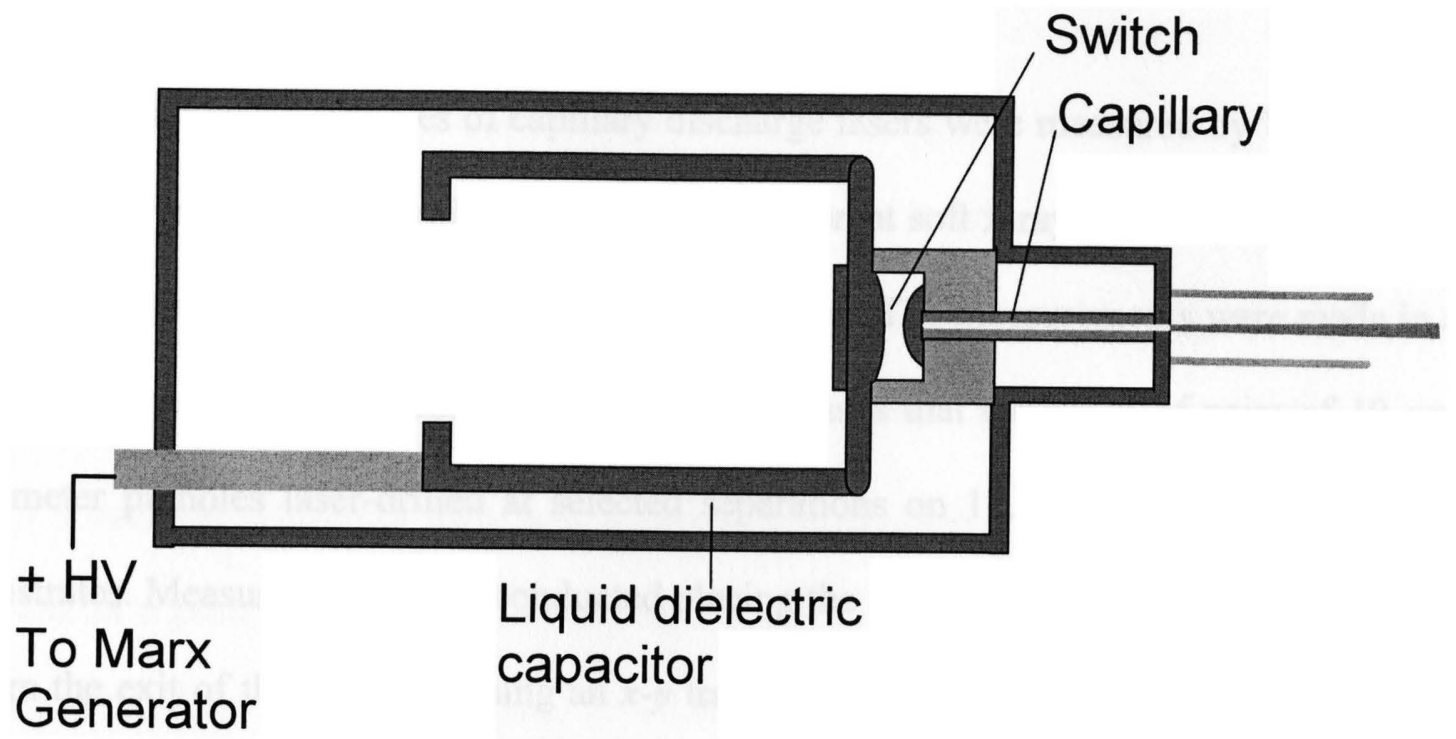

Fig 1.8. Schematic diagram of a table-top capillary discharge laser based on a water dielectric capacitor. The liquid dielectric capacitor is charged to $>200 \mathrm{kV}$.

required the use of a very large Marx generator that occupied a space equivalent to a large fraction of a 4" x 12 " optical table

The next step in the evolution of practical capillary discharge soft $\mathrm{x}$-ray lasers consisted in the reduction of the size and increase in the repetition rate. This was accomplished by B.R. Benware et al. in 1998 by optimization of the discharge design [1.23]. A lower inductance design allowed for sufficiently high current pulses to be generated using a smaller water capacitor with a voltage of $200-250 \mathrm{kV}$. This design fit onto a fraction of a standard optical table with the exception of the Marx generator, which was about twice the size of the power supply of a $1 \mathrm{~J}, 10 \mathrm{~Hz}$ YAG laser. The soft x-ray laser operated up to $10 \mathrm{~Hz}$ and had an average output power of $\sim 1 \mathrm{~mW}$. This increased the average power available from soft x-ray lasers by 2 to 3 orders of magnitude. In 1999 , Macchietto et al. further improved the output of these lasers by demonstrating $\sim 3 \mathrm{~mW}$ 
average power using a $36 \mathrm{~cm}$ long capillary excited by current pulses of $25 \mathrm{kA}$ peak amplitude [1.24].

The coherence properties of capillary discharge lasers were measured by Y. Liu et al. They observed that an essentially full spatially coherent soft x-ray beam can be generated from a capillary discharge laser operating at $46.9 \mathrm{~nm}$. The measurements were made in a Young's interference experiment using pinhole masks that consisted of pairs of $10 \mu \mathrm{m}$ diameter pinholes laser-drilled at selected separations on $12.5 \mu \mathrm{m}$ thick stainless-steel substrates. Measurements were conducted placing the masks at distances of 15 and $40 \mathrm{~cm}$ from the exit of the capillary using an $x-y$ translation stage to position the pinholes with respect to the beam. The interference patterns were recorded with CCD camera at $300 \mathrm{~cm}$ from the mask (see fig. 1.9a). The spatial coherence was observed to improve as a function of capillary length, and a nearly fully coherent beam is emitted from the use of a $36 \mathrm{~cm}$ long capillary, illustrated by the high fringe modulation in figure $1.9 \mathrm{~b}[1.26]$.

The progress on practical soft $\mathrm{x}$-ray lasers is now driven by the development of easily transportable, compact high repetition rate lasers for applications. This is the main goal of the work conducted as part of this thesis, and is described in Chapter 2.

\subsection{Examples of applications of soft $x$-ray lasers}

Applications which require a coherent short wavelength source are numerous and include many scientific disciplines including physics, chemistry and biology. A significant portion of the interest in compact soft $\mathrm{x}$-ray lasers arises from the applications mentioned in section 1.3.2. A description of a few of these applications follows.

\subsubsection{Soft X-ray laser interferometry of dense plasmas}

The ability to produce time-resolved two-dimensional maps of the electron density in 
dense plasmas is of major interest in understanding their dynamics, and validating and improving complex numerical plasma hydrodynamic codes. As discussed, collisionally pumped table-top soft x-ray lasers operate with a high spatial coherence and high peak brightness making it possible to use them for plasma interferometry and other plasma diagnostic techniques. The critical electron density in a plasma scales as $\mathrm{n}_{\mathrm{cr}}=1.110^{21} * \lambda^{-2}$ $\left(\mathrm{cm}^{-3}\right)$ illustrating that shorter wavelengths can probe denser plasmas. Moreno et al. have used a tabletop soft-x-ray laser and a wave-front division interferometer to probe the plasma of a pinch discharge [1.60]. In their experiments a compact capillary dischargepumped Ne-like Ar laser emitting at $46.9 \mathrm{~nm}$ was combined with a wave division interferometer based on Lloyd's mirror and imaging Sc-Si multilayer-coated optics to map the electron density of the discharge demonstrating the feasibility of using table-top soft x-ray lasers for plasma diagnostics. The resolution of interferograms was improved upon when Filevich et al. reported the demonstration of an amplitude-division soft-x-ray interferometer capable of operating at the wavelength of any saturated soft-x-ray laser $(5.6-46.9 \mathrm{~nm})$ that is available at the present time [1.61]. The interferometer, which utilizes grazing-incidence diffraction gratings as beam splitters in a modified MachZehnder configuration, was used in combination with a tabletop $46.9-\mathrm{nm}$ laser to probe a large scale ( 2.7-mm-long) laser-created plasma. This experiment was expanded by Smith et al. to picosecond duration and shorter wavelength of the $14.7 \mathrm{~nm} \mathrm{Ni-like} \mathrm{Pd}$ laser [1.62]. This enabled direct measurement of the electron-density profile to within 10 $\mu \mathrm{m}$ of the target surface. Several other demonstrations of plasma diagnostics using soft $\mathrm{x}$ ray lasers can be found in the literature and references [[1.49-1.50, 1.63-1.64]. 
a)

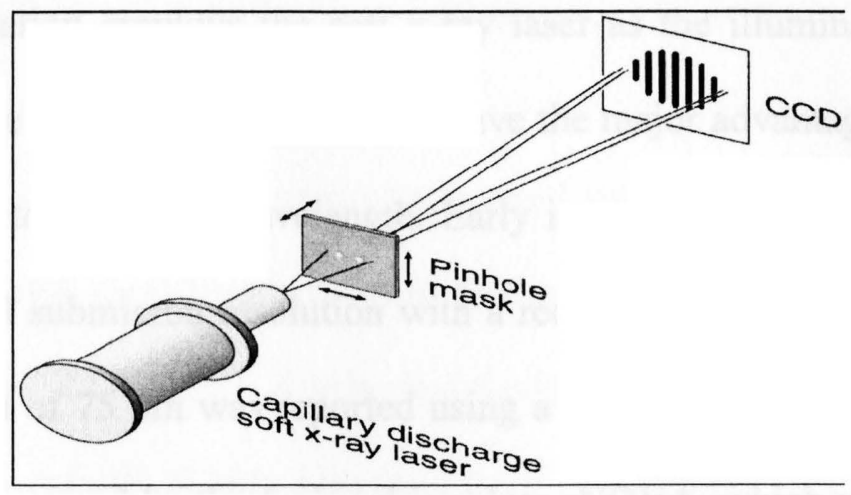

b)
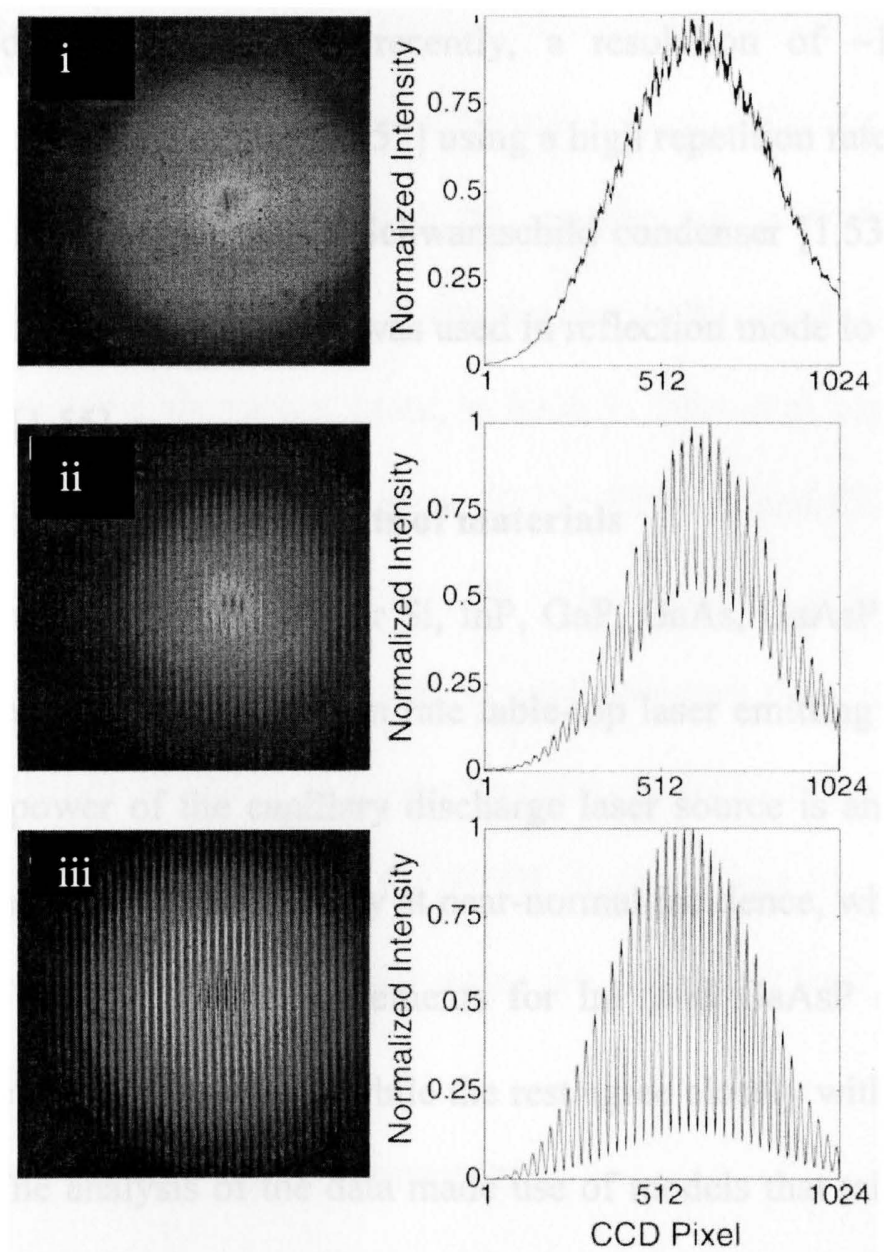

Figure 1.9: a) Schematic representation of the experimental setup used in the two-pinhole interference coherence measurements by Y. Liu et al. [1.26]. b) Interferograms and their lineouts showing the coherence buildup of the laser beam with increasing capillary length. The capillary lengths are (i) 18 , (ii) 27 , and (iii) $36 \mathrm{~cm}$. The figure is taken from reference 1.26 . 


\subsubsection{Soft $x$-ray imaging}

Another application employs the soft $\mathrm{x}$-ray laser as the illumination source for an imaging microscope. Soft x-ray microscopes have the major advantage of resolving very small features due to the short wavelength. Early imaging work with short wavelength lasers demonstrated submicron resolution with a recombination laser $(\lambda=18.2 \mathrm{~nm})[1.4]$. A higher resolution of $75 \mathrm{~nm}$ was reported using a very large size collisional soft $\mathrm{x}$-ray laser $(\lambda=4.48 \mathrm{~nm})$ pumped by the fusion driver laser NOVA, which was limited to firing several shots a day [1.51]. More recently, a resolution of $\sim 120-150 \mathrm{~nm}$, was demonstrated by G. Vaschenko et al. [1.52] using a high repetition rate $46.9 \mathrm{~nm}$ capillary discharge laser in combination with a Schwarzschild condenser [1.53] and a zone plate objective [1.54]. The same microscope was used in reflection mode to display features as small as $\sim 100 \mathrm{~nm}[1.55]$.

\subsubsection{Determination of optical constants of materials}

Optical constants were measured for Si, InP, GaP, GaAs, GaAsP, and Ir utilizing a reflectrometer based on a high repetition rate table-top laser emitting at $46.9 \mathrm{~nm}$ [1.56]. The high average power of the capillary discharge laser source is an advantage for the accurate measurements of the reflectivity at near-normal incidence, where the reflectivity of most materials is low. The measurements for InP and GaAsP constitute the first experimental value at this wavelength while the rest agree closely with values previously tabulated [1.59]. The analysis of the data made use of models that take into account the presence of a surface layer of contaminants. Comparison of the values of the optical constants obtained from having surface layers of different characteristics shows that the 
optical constants of the bulk material obtained with this treatment remain unchanged even when the characteristics of the surface layer changes significantly.

\subsubsection{Soft $x$-ray laser ablation}

The generation and characterization of a focused soft-x-ray laser beam with intensity and energy density that significantly exceed the threshold for the ablation of metals was reported by Benware et al. [1.57]. A spherical Sc-Si multilayer mirror was used to focus high-energy laser pulses $(0.13 \mathrm{~mJ})$ generated by a 46.9 -nm tabletop capillary-discharge amplifier, yielding the first clear demonstration of material ablation with a coherent soft x-ray beam. The focused laser beam was observed to have sufficient intensity to ablate aluminum, stainless steel and brass when the samples are positioned within several hundred $\mu \mathrm{m}$ from the focus. The peak intensities are estimated to be $\sim 110^{11} \mathrm{~W} / \mathrm{cm}^{2}$, dominated by the spherical aberration. Later, in 2005, L. Juha et al. report on the ablation behavior of three common organic polymers: PTFE, PMMA, and PI, irradiated with an intense focused $46.9 \mathrm{~nm}$ laser beam [1.58]. The ablation processes induced by nanosecond pulses of $46.9 \mathrm{~nm}$ laser radiation are compared with conventional longer wavelength laser sources. Contrary to longer wavelength ablation, using a compact 46.9 nm results show similar ablation rates for PTFE, PMMA, and PI. This uniformity of ablation rates could be very beneficial for using the soft x-ray laser radiation in direct polymer nanostructuring [1.58].

\subsubsection{Soft $x$-ray applications to photochemistry}

Additionally, many applications in photophysics and photochemistry would benefit from the use of a compact soft x-ray laser. The capillary discharge laser that is the topic of this thesis was designed to be used as a single photon photoionization source in time of 
flight mass spectroscopy experiments for the study of metal-oxide nanoclusters. The single photon ionization events made possible by the high photon energy greatly reduces negative effects such as fragmentation of the molecules leading to loss of parent/ion information observed in multiphoton ionization experiments. Also, inner shell electrons become accessible with soft $\mathrm{x}$-ray radiation. The single photon ionization of molecules is an important part of the work in this thesis and is presented in great detail in Chapter 3.

\subsection{Summary}

Numerous applications spanning many different fields of study require the use of short wavelength lasers. It is this need that motivates the development of more compact soft x-ray sources that can be implemented in a standard university or industrial laboratory. Through examples of applications, this chapter has established the motivation for the development of a very compact high repetition rate soft x-ray laser. Capillary discharges allow the creation of plasmas with the characteristics required to explore the amplification of soft $\mathrm{x}$-ray radiation. The rest of this thesis discusses the development of transportable $46.9 \mathrm{~nm}$ laser source and its use as a photoionization source for nanocluster mass spectroscopy experiments. 


\section{References}

[1.1] D.L. Matthews, P.L. Hagelstein, M.D. Rosen, M.J. Eckart, N.M. Ceglio, A.U. Hazi, H. Medecki, B.J. MacGowan, J.E. Trebes, B.L. Whitten, E.M. Campbell, C.W. Hatcher, A.M. Hawryluk, R.L. Kauffman, L.D. Pleasance, G. Rambach, J.H. Scofield, G. Stone, T.A. Weaver, "Demonstration of a Soft X-Ray Amplifier," Phys. Rev. Lett. 54, 110 (1985).

[1.2] B.J. MacGowan, L. B. Da Silva, D. J. Fields, C. J. Keane, J. A. Koch, R. A. London, D. L. Matthews, S. Maxon, S. Mrowka, A. L. Osterheld, J. H. Scofield, G. Shimkaveg, J. E. Trebes, and R. S. Walling, "Short wavelength X-ray laser research at the Lawrence Livermore National Laboratory," Phys. Fluids B 4, 2326, (1992); A. Carrillon et al. H.Z. Chen, P. Dhez, L. Dwivedi, J. Jacoby, P. Jaegle, G. Jamelot, J. Zhang, M.H. Key, A. Kidd, A. Klishnick, R. Kodama, J. Krishnan, C.L.S. Lewis, D. Neely, P. Norreys, D. Oneill, G.J. Pert, S.A. Ramsden, J.P. Raucourt, G.J. Tallents, J. Uhomoibhi, "Saturated and near-diffraction-limited operation of an XUV laser at $23.6 \mathrm{~nm}$," Phys. Rev. Lett. 68, 2917, (1992).

[1.3] J.J. Rocca, V.N. Shlyaptsev, F.G. Tomasel, O.D. Cortazar, D. Hartshorn, and J.L.A. Chilla, "Demonstration of a Discharge Pumped Table-Top Soft-X-Ray Laser," Phys. Rev. Lett., 73, 2192 (1994).

[1.4] J. Dunn, Y. Li, A. L. Osterheld, J. Nilsen, J. R. Hunter, V. N. Shlyaptsev, "Gain Saturation Regime for Laser-Driven Tabletop, Transient Ni-Like Ion X-Ray Lasers," Phys. Rev. Lett. 84, 4834 (2000)

[1.5] S. Sebban,. R. Haroutunian, Ph. Balcou, et al., Phys. Rev. Lett. 86, 3004 (2001) and S. Sebban, T. Mocek, D. Ross, et al., "Demonstration of a Ni-Like Kr Optical-FieldIonization Collisional Soft X-Ray Laser at 32.8 nm," Phys. Rev. Lett. 89, 253901 (2002).

[1.6] K.A. Jenulewicz, A. Lucianetti, G. Pruebe, W. Sadner and P.V. Nickles, "Saturated Ni-like Ag x-ray laser at $13.9 \mathrm{~nm}$ pumped by a single picosecond laser pulse," Phys. Rev. A 68, 051802 (2003).

[1.7] A. Butler, A.J. Gonsalves, C.M. McKenna, D.J. Spence, S.M. Hooker, S. Sebban, T. Mocek, I. Betttaibi and B. Cros, "41.8-nm Xe ${ }^{8+}$ laser driven in a plasma waveguide," Phys. Rev. A 70, 023821 (2004).

[1.8] D. Attwood, "Soft X-rays and Extreme Ultraviolet Radiation: Principles and Applications," (Cambridge Univ. Press, 1999).

[1.9] S. Suckewer, C.H. Skinner, H. Milchberg, C. Keane, and D. Voorhees, "Amplification of stimulated soft x-ray emission in a confined plasma column," Phys. Rev. Lett. 55, 1753 (1985). 
[1.10] J. J. Rocca, “Table-top soft x-ray lasers,” Rev. Sci. Inst. 70, 3799 (1999).

[1.11] M. Born and E. Wolf, "Principles of Optics," (Pergamon, New York, 1983), Chapter 10; For a more advanced discussion see L. Mandel and E. Wolf, "Optical Coherence and Quantum Optics," (Cambridge Univ. Press, 1995).

[1.12] J.W. Goodman, "Statistical Optics," (Wiley, New York, 1985).

[1.13] P.H. van Cittert, "Die Wahrscheinliche Schwingungsverteilung in Einer von Einer Lichtquelle Direkt oder Mittls Einer Linse Beleuchteten Ebene" [The Probable Distribution of Vibrations in a Plane Illuminated by a Light Source Either Directly or Through a Lens], Physica 1, 210 (1934).

[1.14] F. Zernike, "The Concept of Degree of Coherence and its Application to Optical Problems," Physica 5, 785 (1938).

[1.15] D. T. Attwood, K. Halbach, and N. J. Kim, Science 228, 1264 (1985).

[1.16] D. T. Attwood, G. Sommargren, R. Beguiristain, K. Nguyen, J. Boker, N. Ceglio, K. Jackson, M. Koike, and J. Underwood, "Undulator radiation for at-wavelength interferometry of optics for extreme-ultraviolet lithography," Appl. Opt. 32, 7022 (1993).

[1.17] R. Coisson, "Spatial coherence of synchrotron radiation," Appl. Opt. 34, 904 (1995).

[1.18] Z. Chang, A. Rundquist, H. Wang, M. M. Murnane, and H. C. Kapteyn, "Generation of Coherent Soft X Rays at $2.7 \mathrm{~nm}$ Using High Harmonics," Phys. Rev. Lett. 79, 2967 (1997).

[1.19] A. Rundquist, C. G. Durfee III, Z. Chang, C. Herne, S. Backus, M. Murnane, and H. C. Kapteyn, "Phase-Matched Generation of Coherent Soft X-rays," Science 280, 1412 (1998).

[1.20] T. Ditmire, J. K. Crane, H. Nyugen, L. B. Da Silva, and M. D. Perry, "Energyyield and conversion-efficiency measurements of high-order harmonic radiation," Phys. Rev. A 51, R902 (1995).

[1.21] J. J. Rocca, V. Shlyaptsev, F.G. Tomasel, O.D. Cortazar, D. Hartshorn, J.L.A. Chilla, "Demonstration of a discharge pumped table-top soft x-ray laser," Phys. Rev. Lett. 73, 2192, (1994).

[1.22] J. J. Rocca, D. P. Clark, J. L. A. Chilla, V. N. Shlyaptsev, "Energy Extraction and Achievement of the Saturation Limit in a Discharge-Pumped Table-Top Soft X-Ray Amplifier," Phys. Rev. Lett. 77, 1476 (1996). 
[1.23] B. R. Benware, C. D. Macchietto, C. H. Moreno, and J. J. Rocca, "Demonstration of a high average power table top soft x-ray laser," Phys. Rev. Lett. 81, 5804 (1998).

[1.24] C. D. Macchietto, B. R. Benware, and J. J. Rocca, "Generation of millijoule-level soft-x-ray laser pulses at a $4-\mathrm{Hz}$ repetition rate in a highly saturated tabletop capillary discharge amplifier," Opt. Lett. 24, 1115 (1999).

[1.25] M.D. Rosen, P.L. Hagelstein, D.L. Matthews, E.M. Campbell, A.U. Hazi, B.L. Whitten, B. MacGowan, R.E. Turner, and R.W. Lee, "Exploding Foil Technique for Achieving a Soft X-ray Laser," Phys. Rev. Lett. 54, 106 (1985).

[1.26] Y. Liu, M. Seminario, F.G. Tomasel, C. Chang,, J.J. Rocca, and D.T. Attwood, "Achievement of essentially full spatial coherence in a high-average-power soft-x-ray laser," Phys. Rev. A 63, 033802-1 (2001).

[1.27] J. Nilsen, B.J. MacGown, L.B. Da Silva, and J.C. Moreno, "Prepulse technique for producing low-Z Ne-like x-ray lasers," Phys. Rev. A 48, 4682 (1993).

[1.28] Y. Li, G. Pretzler, E Fill, and J. Nilsen, "Temporally and spatially resolved investigation of the $J=0-1$ and $J=2-1,3 p-3 s$ laser emissions in neonlike germanium," J. Opt. Soc. Am. B 13, 742 (1996).

[1.29] M.A. Larotonda, B.M. Luther, Y. Wang, Y. Liu, D. Alessi, M. Berrill, A. Dummer, F. Brizuela, C.S. Menoni, M.C. Marconi, V.N. Shlyaptsev, J. Dunn, and J.J. Rocca, "Characteristics of a Saturated 18.9-nm Tabletop Laser Operating at $5-\mathrm{Hz}$ Repetition Rate," IEEE J. of Selected Topics in Q. Electronicd 10, 1363 (2004).

[1.30] P.V. Nickles, V.N. Shlyaptsev, M. Kalachnikov, M. Schnurer, I. Will, and W. Sandner, "Short Pulse X-Ray Laser at $32.6 \mathrm{~nm}$ Based on Transient Gain in Ne-like Titanium," Phys. Rev. Lett. 78, 2748 (1997).

[1.31] S. Sebban, L. M. Upcraft, P. Balcou, M. Pittman, R. Haroutunian, G. Grillon, C. Valentin, A. Rousse, J. P. Rousseau, L. Notebaert, and A. D. Hulin, "Investigations on collisionally pumped optical field ionization soft X-ray lasers," J. Opt. Soc. Amer. B 20, 195 (2003).

[1.32] P. B. Corkum and N. H. Burnett, "Multiphoton ionization for the production of $x-$ ray laser plasmas," in Short Wavelength Coherent Radiation, R. W. Falcone and J. Kirtz, OSA Proceedings Series 2, 225 (1988).

[1.33] P. B. Corkum, N. H. Burnett, and F. Brunel, "Above threshold ionization in the long-wavelength limit," Phys. Rev. Lett. 62, 1259 (1989).

[1.34] S. Sebban, R. Haroutunian, P. Balcou, G. Grillon, A. Rousse, S. Kazamias, T. 
Marin, J. P. Rousseau, L. Notebaert, M. Pittman, J. P. Chambaret, A. Antonetti, D. Hulin, D. Ros, A. Klisnick, A. Carillon, P. Jaeglé, G. Jamelot, and J. F. Wyart, "Saturated amplification of a collisionally pumped optical field ionization soft X-ray laser at 41.8 nm," Phys. Rev. Lett. 86, 3004 (2001).

[1.35] S. Sebban, T. Mocek, D. Ros, L. Upcraft, P. Balcou, R. Haroutunian, G. Grillon, B. Rus, A. Klisnick, A. Carillon, G. Jamelot, C. Valentin, A. Rousse, J. P. Rousseau, L. Notebaert, M. Pittman, and A. D. Hulin, "Demonstration of a Ni-like $\mathrm{Kr}$ optical-fieldionization collisional soft X-ray laser at $32.8 \mathrm{~nm}$," Phys. Rev. Lett. 89, 253 (2002).

[1.36] Y. Nagata, K. Midorikawa, S. Kubodera, M. Obara, H. Tashiro, and K. Tokoda, "Soft-x-ray amplification of the Lyman- alpha transition by optical-field-induced ionization," Phys. Rev. Lett. 71, 3774 (1993).

[1.37] D. Korobkin, C. H. Nam, S. Suckewer, and A. Golstov, "Demonstration of Soft XRay Lasing to Ground State in Li III," Phys. Rev. Lett. 77, 5206 (1996).

[1.38] J.J. Rocca, D.P. Clark, J.L.A. Chilla and V.N. Shlyaptsev, "Energy Extraction and Achievement of the Saturation Limit in a Discharge-Pumped Table-Top Soft X-Ray Amplifier," Phys. Rev. Lett., 77, 1476 (1996).

[1.39] R. Vesey, A. Campbell, B. Robert, S.A. Hanson, D. Lester, M. Edward, T. Alan, Porter, J. Larry, "Z-pinch driven fast ignition fusion,".Sandia National Laboratories, Albuquerque, NM (2004).

[1.40] A. Ben-Kish, M. Shuker, R.A. Nemirowsky, A. Ron, and J.L. Schwob, "Plasma Dynamics in Capillary Discharge Soft X-Ray Lasers," Phys. Rev. Lett. 87, 1 (2001).

[1.41] A. Ritucci, G. Tomassetti, A. Reale, L. Palladino, L. Reale, F. Flora, L. Mezi, S.V. Kukhlevsky., A. Faenov, T. Pikuz, "Investigation of a highly saturated soft X-ray amplification in a capillary discharge plasma waveguide," Applied Phys. B 78, 965 (2004).

[1.42] J. Filevich, K. Kanizay, M.C. Marconi, J.L.A. Chilla, and J.J. Rocca., "Dense plasma diagnostics with an amplitude-division soft-x-ray laser interferometer based on diffraction gratings," Optics Lett. 25, 356 (2000).

[1.43] A. Artioukov, B.R. Benware, J.J. Rocca, M. Forsythe, Y.A. Uspenskii, A.V. Vinogradov, "Determination of XUV optical constants by reflectometry using a highrepetition rate 46.9-nm laser," IEEE J. of Selected Topics in Quant. Elec. 5, 1495 (1999). 
[1.44] B.R. Benware, A. Ozols, J.J. Rocca, I.A. Artioukov, V.V. Kondratenko and A.V. Vinogradov, "Focusing of a tabletop sof t-x-ray laser beam and laser ablation," Optics Lett. 24, 1714 (1999).

[1.45] M. Seminario, J.J. Rocca, R. Depine, B. Bach, and B. Bach, "Characterization of Diffraction Gratings by use of a Tabletop Soft-X-Ray Laser," Appl. Optics 40, 5539 (2001).

[1.46] M.G. Capeluto, G. Vaschenko, M. Grisham, M.C. Marconi et al., "Nanopatterning with interferometric lithograph using a compact $\lambda=46.9 \mathrm{~nm}$ laser," (submitted to IEEE Transac. on Nanotechnology).

[1.47] A. Endo, "High-Average Power EUV Light Source for the Next-Generation Lithography by Laser-Produced Plasma," IEEE J. of Selected Topics in Quant. Elec. 10, $6,(2004)$.

[1.48] L.B. Da Silva, T.W. Barbee, Jr., R. Cauble, P. Celliers, D. Ciarlo, S. Libby, R.A. London, D. Matthews, S. Mrowka, J.C. Moreno, D. Ress, J.E. Trebes, A.S. Wan, F. Weber, "Electron Density Measurements of High Density Plasmas Using Soft X-Ray Laser Interferometry," Phys. Rev. Lett. 74, 3991, (1995).

[1.49] M. C. Marconi, C. H. Moreno, J. J. Rocca, V. N. Shlyaptsev, A. L. Osterheld, "Dynamics of a microcapillary discharge plasma using a soft x-ray laser backlighter," Phys. Rev. E 62, 19675 (2000).

[1.50] J. Filevich, J. J. Rocca, M. C. Marconi, S. J. Moon, J. Nilsen, J. H. Scofield, J. Dunn, R. F. Smith, R. Keenan, J. R. Hunter, V. N. Shlyaptsev, "Observation of a Multiply Ionized Plasma with Index of Refraction Greater than One," Phys. Rev. Lett. 94, 035005 (2005)

[1.51] L. Da Silva et al., Science 258, 269 (1992). WHICH is THE PAGE nuMBLR AND WHCH IS THE VOLUME?

[1.52] G. Vaschenko, F. Brizuela, C. Brewer, M. Grisham, H. Mancini, C.S. Menoni, M. Marconi, and J. J. Rocca, "Nano-imaging with a compact extreme ultraviolet laser," Opt. Lett. in press (2005).

[1.53] I. A. Artioukov, A. V. Vinogradov, V. E. Asadchikov, Y. S. Kasyanov, R. V. Serov, A. I. Fedorenko, V. V. Kondratenko, and S. Y. Yulin, "Schwartzschild soft-x-ray microscope for imaging of nonradiating objects," Opt. Lett. 20, 2451-2453 (1995).

[1.54] W. Chao, E. Anderson, G. P. Denbeaux, B. Harteneck, J. A. Liddle, D. L. Olynick, A. L. Pearson, F. Salmassi, C. Y. Song, and D. T. Attwood, "20-nm-resolution soft X-ray microscopy demonstrated by use of multilayer test structures," Opt. Lett. 28, 2019-2021 (2003). 
[1.55] F. Brizuela, G. Vaschenko, C. Brewer, M. Grisham, C. S. Menoni, M. C. Marconi, J. J. Rocca, W. Chao, J. A. Liddle, E. H. Anderson, D. T. Attwood, A. V. Vinogradov, I. A. Artioukov, Y. P. Pershyn and V. V. Kondratenko, "Reflection mode imaging with nanoscale resolution using a compact extreme ultraviolet laser," Optics Express 13, 3983 (2005).

[1.56] I.A. Artioukov, B.R. Benware, J.J. Rocca, M. Forsythe, Y.A. Uspenskii, A.V. Vinogradov, "Determination of XUV optical constants by reflectometry using a highrepetition rate 46.9-nm laser", IEEE Journal Of Selected Topics In Quantum Electronics 5, 1495 (1999).

[1.57] B.R. Benware, A. Ozols, J.J. Rocca, I.A. Artioukov, V.V. Kondratenko and A.V. Vinogradov, "Focusing of a soft x-ray laser beam and laser ablation", Optics Lett. 24, 1714 (1999).

[1.58] L. Juha, M. Bittner, D. Chvostova, J. Krasa, Z. Otcenasek, A.R. Prag, J.

Ullschmied, Z. Pientka, J. Krzywinski, J.B. Pelka, A. Wawro, M.E. Grisham, G.O.

Vaschenko, C.S. Menoni, J.J. Rocca, "Ablation of organic polymers by 46.9-nm-laser radiation”, Applied Physics Letters 86, 034109, (2005).

[1.59] E.D. Palik, Handbook of Optical Constants of Solids, Academic Press, San Diego, CA, 1998.

[1.60] C.H. Moreno, M.C.Marconi, K. Kanizay, J.J. Rocca, Y.A. Uspenskii, A.V. Vinogradov and Y.A. Pershin, "Soft x-ray laser interferometry of a pinch discharge using a table-top laser," Phys. Rev. E 60, 911 (1999).

[1.61] J. Filevich, K. Kanizay, M.C. Marconi, J.L.A. Chilla, and J.J. Rocca, "Dense plasma diagnostics with an amplitude division soft $\mathrm{x}$-ray laser interferometer based on diffraction gratings", Optics Lett. 25, 356 (2000).

[1.62] R.F. Smith, J.Dunn, J. Nilsen, V.N. Shlyaptsev, S. Moon, J. Filevich, J.J. Rocca, M.C. Marconi, J.R. Hunter, and T.W. Barbee, Jr., "Picosecond X-ray laser interferometry of dense plasmas," Physical Review Letters 89, 065004 (2002).

[1.63] J. Filevich, J.J. Rocca, E. Jankowska, E.C. Hammarsten, M.C. Marconi, S.Moon and V.N. Shlyaptsev, "Two-dimensional effects in laser-created plasmas measured with soft-x-ray laser interferometry", Physical Review E 67, (2003).

[1.64] J. Filevich, J.J. Rocca, M.C. Marconi, R.F. Smith, J. Dunn, R. Keenan, J.R. Hunter, S.J. Moon, J. Nilsen, A. Ng, V.N. Shlyaptsev, "Picosecond resolution soft X-ray laser plasma interferometry", Applied Optics 43, 3938 (2004). 
[1.65] B.E. Lemoff, G.Y. Yin, C.L. Gordon III, C.P.J. Barty, and S.E. Harris, "Demonstration of a 10-Hz Femtosecond-Pulse-Driven XUV Laser at $41.8 \mathrm{~nm}$ in Xe IX," Phys. Rev. Lett. 74, 1574 (1995).

[1.66] M.A. Larotonda, B.M Luther, Y. Wang, Y. Liu, D. Alessi, M. Berrill, A. Dummer, F. Brizuela, C.S. Menoni, M.C. Marconi, V.N. Shlyaptsev, J. Dunn, and J.J. Rocca, "Characteristics of a saturated $18.9-\mathrm{nm}$ tabletop laser operating at $5-\mathrm{Hz}$ repetition rate," IEEE Trans. on Plasma Sci. 10, 1363 (2005).

[1.67] R. Tommasini, J. Nilsen, and E. E. Fill, Proc. SPIE 4505, 85 (2001).

[1.68] T. Ozaki, R. A. Ganeev, A. Ishizawa, T. Kanai, and H. Kuroda, Phys. Rev. Lett. 89, 253902 (2002); R. Li and Z. Z. Xu, J. Phys. IV 11_PR2_, 27 (2001).

[1.69] V. N. Shlyaptsev, J. Dunn, S. Moon, R. Smith, R. Keenan, J. Nilsen, K. B. Fournier, J. Kuba, A. L. Osterheld, J. J. Rocca, B. Luther, Y. Wang, and M. Marconi, Proc. SPIE 5197, 221 (2003).

[1.70] R. Keenan, J. Dunn, P. K. Patel, D. F. Price, R. F. Smith, and V.N. Shlyaptsev, Phys. Rev. Lett. 94, 103901 (2005); R. Keenan, J. Dunn, V. N. Shlyaptsev, R. Smith, P. K. Patel, and D. F. Price, Proc. SPIE 5197, 213 (2003).

[1.71] B. M. Luther, Y. Wang, M. A. Larotonda, D. Alessi, M. Berrill, M. C. Marconi, V. N. Shlyaptsev, and J. J. Rocca, Opt. Lett. 30, 165 (2005).

[1.72] D. Alessi, B. M. Luther, Y. Wang, M. A. Larotonda, M. Berrill, and J. J. Rocca, Opt. Express 13, 2093 (2005).

[1.73] Y. Wang, M. A. Larotonda, B. M. Luther, D. Alessi, M. Berrill, V. N. Shlyaptsev, and J. J. Rocca, "Demonstration of high-repetition-rate tabletop soft-x-ray lasers with saturated output at wavelengths down to $13.9 \mathrm{~nm}$ and gain down to $10.9 \mathrm{~nm}$," Phys. Rev. A 72, 053807 (2005). 


\section{CHAPTER 2}

\section{DESIGN AND OPERATION CHARACTERISTICS OF HIGH REPETITION RATE DESK-TOP SIZE 46.9 nm CAPILLARY DISCHARGE LASER}

In this chapter, the design and demonstration of a desk-top size high repetition rate capillary discharge-pumped soft x-ray laser is covered. The laser was developed with the objective of realizing a high repetition rate single-photon photoionization source for photochemistry applications, an application discussed in Chapter 3. The first section focuses mainly on the compact desk-top design and the discharge parameters required for operation of the laser. The remaining sections will present the measurements taken to characterize the operation of the laser at repetition rates up to $12 \mathrm{~Hz}$. Observations on the lifetime of the capillary will also be discussed.

\subsection{Design Characteristics of a Desk-top Size High Repetition Rate Capillary Discharge-pumped Soft X-ray Laser}

We describe a capillary discharge laser that is significantly more compact and less costly than its predecessors. It is to our knowledge the first soft x-ray laser to fit onto a small desk, and to be easily transportable (Fig 2.1b), and also the first such laser to be installed in a photochemistry lab and used as a photoionization source in time of flight mass spectroscopy (TOFMS) systems. The laser emits $>10 \mu \mathrm{J}$ pulses of $\lambda=46.9 \mathrm{~nm}$ light at up to $12 \mathrm{~Hz}$ repetition rate. It occupies a table area of approximately $0.4 \times 0.4 \mathrm{~m}^{2}\left(0.4 \times 0.8 \mathrm{~m}^{2}\right.$ including the vacuum pump), smaller than that occupied by many widely used ultraviolet gas lasers. The power supplies and gas handling system can be accommodated in two small racks that fit under a standard optical table. The reduced size of this capillary discharge device is achieved making use of a very low inductance co-axial discharge configuration (Fig. 2.1a) 

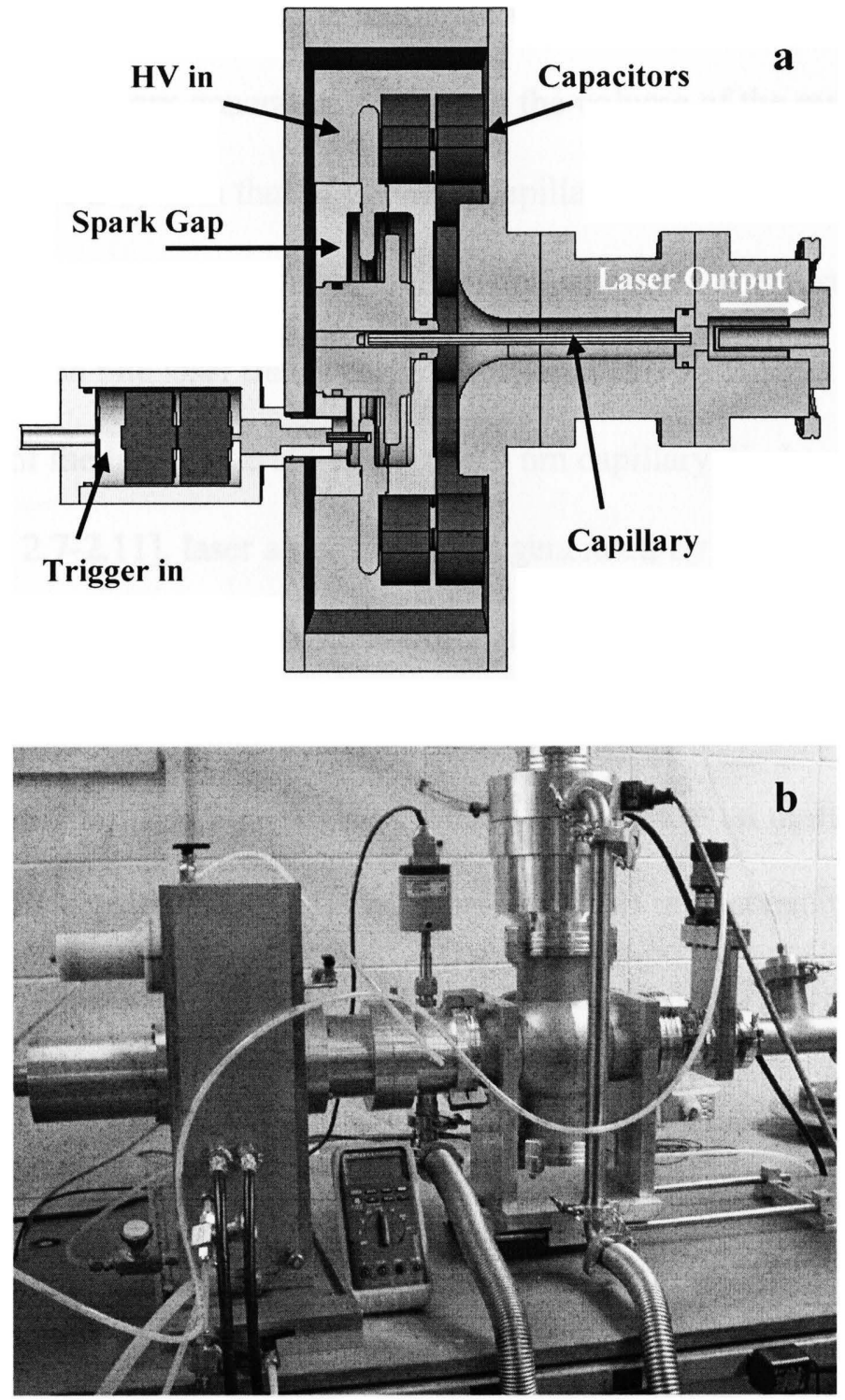

Fig. 2.1. (a) Schematic representation of the $46.9 \mathrm{~nm}$ capillary discharge laser. (b) photograph of the laser device. A handheld multimeter is shown to provide a reference of scale. 
that decreases the voltage necessary to generate the peak current required for laser excitation. This allows the excitation of the capillary discharge channel utilizing strontium titanate capacitors, which are charged to moderate voltages $(<90 \mathrm{kV})$. The reduced voltage eliminates the need of a Marx generator. As a result the volume of the pulsed power unit is 9 times smaller (figure 2.2) than that of previous capillary discharge lasers [2.1] and can be accommodated in a small rack under a regular optical table. The result is an extremely compact high repetition rate laser that is easily transportable (Fig. $2.1 \mathrm{~b}$ ).

As in the case of the larger size Ne-like Ar $46.9 \mathrm{~nm}$ capillary discharge lasers previously demonstrated [2.2, 2.7-2.11], laser amplification is generated by fast discharge excitation of an Ar-filled capillary tube. The magnetic force of the current pulse and large thermal pressure gradients near the wall rapidly compress the plasma to form a dense and hot column with a large density of Ne-like ions, with a very high axial uniformity and a length to diameter ratio of the order of 1000:1. Collisional electron impact excitation of the ground state Ne-like ions produces a population inversion between the $3 \mathrm{p}^{1} \mathrm{~S}_{0}$ and $3 \mathrm{~s}{ }^{1} \mathrm{P}_{1}$ levels, resulting in amplification at $46.9 \mathrm{~nm}$ [2.6].

Laser amplification is obtained in a plasma column generated in an aluminum-oxide capillary $3.2 \mathrm{~mm}$ inside diameter and $21 \mathrm{~cm}$ in length filled with pre-ionized Ar gas at an optimized pressure of 700 mTorr. The plasma column is excited by current pulses of $\approx 22$ kA peak amplitude that are monitored with a Rogowski coil. The excitation current pulse is produced by discharging a set of strontium titanate capacitors with a combined capacitance of $27 \mathrm{nF}$ through a high voltage spark-gap switch pressurized by air (main spark-gap) that is 


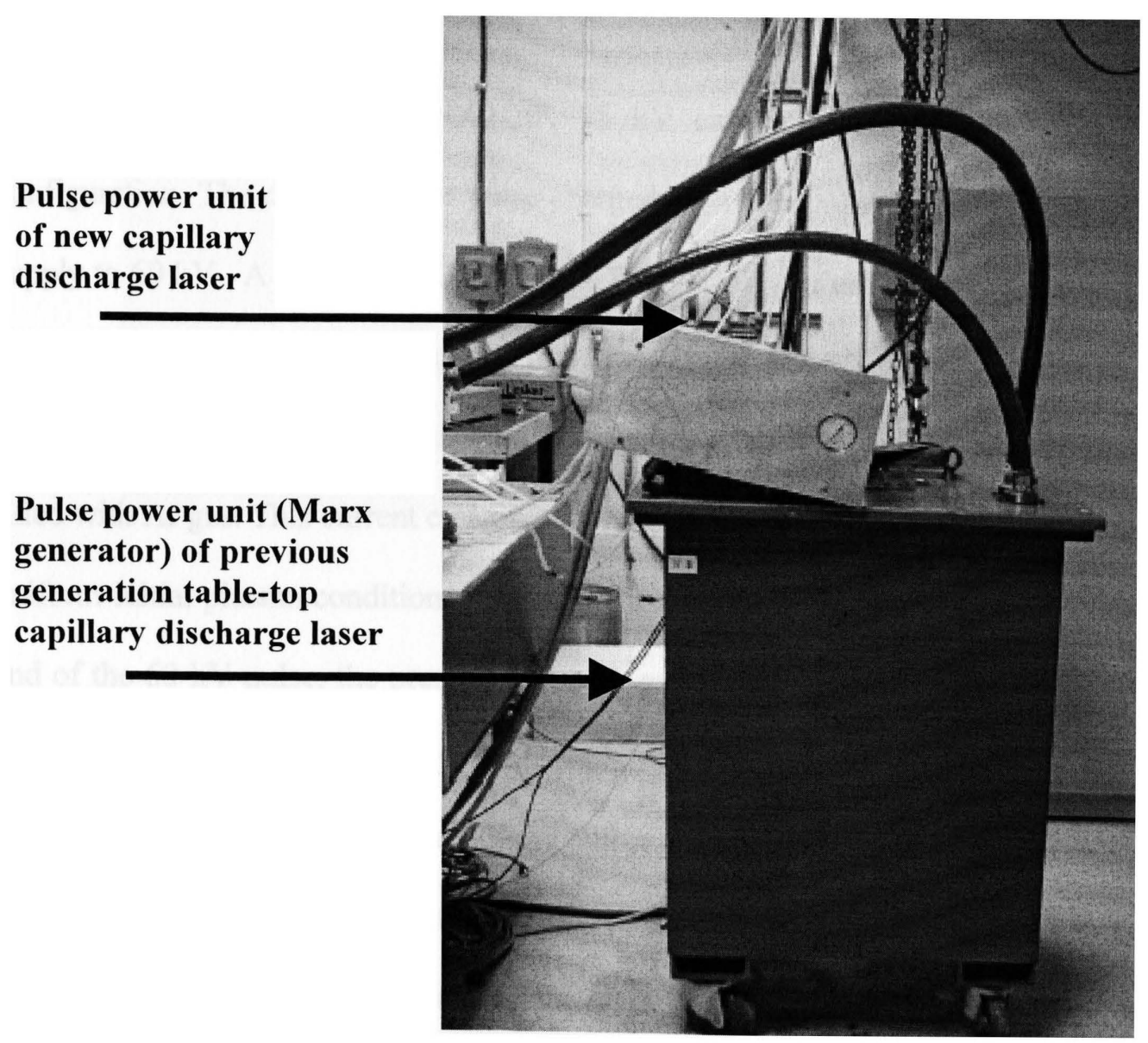

Fig. 2.2: Size comparison of pulsed power units for new and old capillary discharge lasers. The new pulse power unit is $\sim 9$ times smaller than the Marx generator. 
connected in series with the capillary load. The capacitors, which are placed in a ring configuration surrounding the main spark-gap, are pulse-charged to $80-90 \mathrm{kV}$ by a pulsed power unit that is enclosed in a separate box and connected to the laser head with a high voltage coaxial cable. Figure 2.3 shows a schematic diagram and a photograph of the single stage pulsed power.

$\mathrm{C}_{1}$ represents a set of six $40 \mathrm{nF}, 35 \mathrm{kV}$ capacitors arranged in a parallel/series configuration. The capacitors are pulse-charged by a General Atomics high voltage power supply to $60 \mathrm{kV}$. A Stanford DG535 digital delay generator enables the power supply with a TTL square pulse that has a duration of $33 \mathrm{~ms}$. The $60 \mathrm{kV}, 33 \mathrm{~ms}$ pulse also appears across resistor $R_{5}$ and creates a preionization current that is directly applied to the capillary load filled with Ar gas. This current creates a lowly ionized state of the Ar gas and helps to create uniform initial plasma conditions necessary for a stable compression. At $100 \mu$ s before the end of the $60 \mathrm{kV}$ pulse, the pressurized spark-gap switch shown in figure $2.3 \mathrm{~b}$ is triggered and rapidly closes. Consequently, the capacitor bank $\left(\mathrm{C}_{1}\right)$ quickly discharges and forms an RLC circuit with $L_{1}, R_{1}$. This causes the output voltage of the pulsed power unit to ring to $\sim 80-90 \mathrm{kV}$ and pulse-charges the ring of ceramic capacitors shown in figure 2.1a. At the same time the spark-gap switch closes, the resistor/capacitor network represented by $\mathrm{C}_{2}, \mathrm{C}_{3}$, $R_{2}$, and $R_{3}$ creates a small current prepulse that increases the ionization level of the Ar preplasma. This further supports the creation of uniform initial plasma conditions.

The final stage of ionization in creating a Ne-like Ar plasma is achieved when the sparkgap shown in figure 2.1a is closed and discharges the ring of ceramic capacitors sending a large current pulse through the capillary load. This main current pulse is initiated by triggering the main spark-gap with a $\sim-50 \mathrm{kV}$ pulse applied to the trigger input also shown 
a

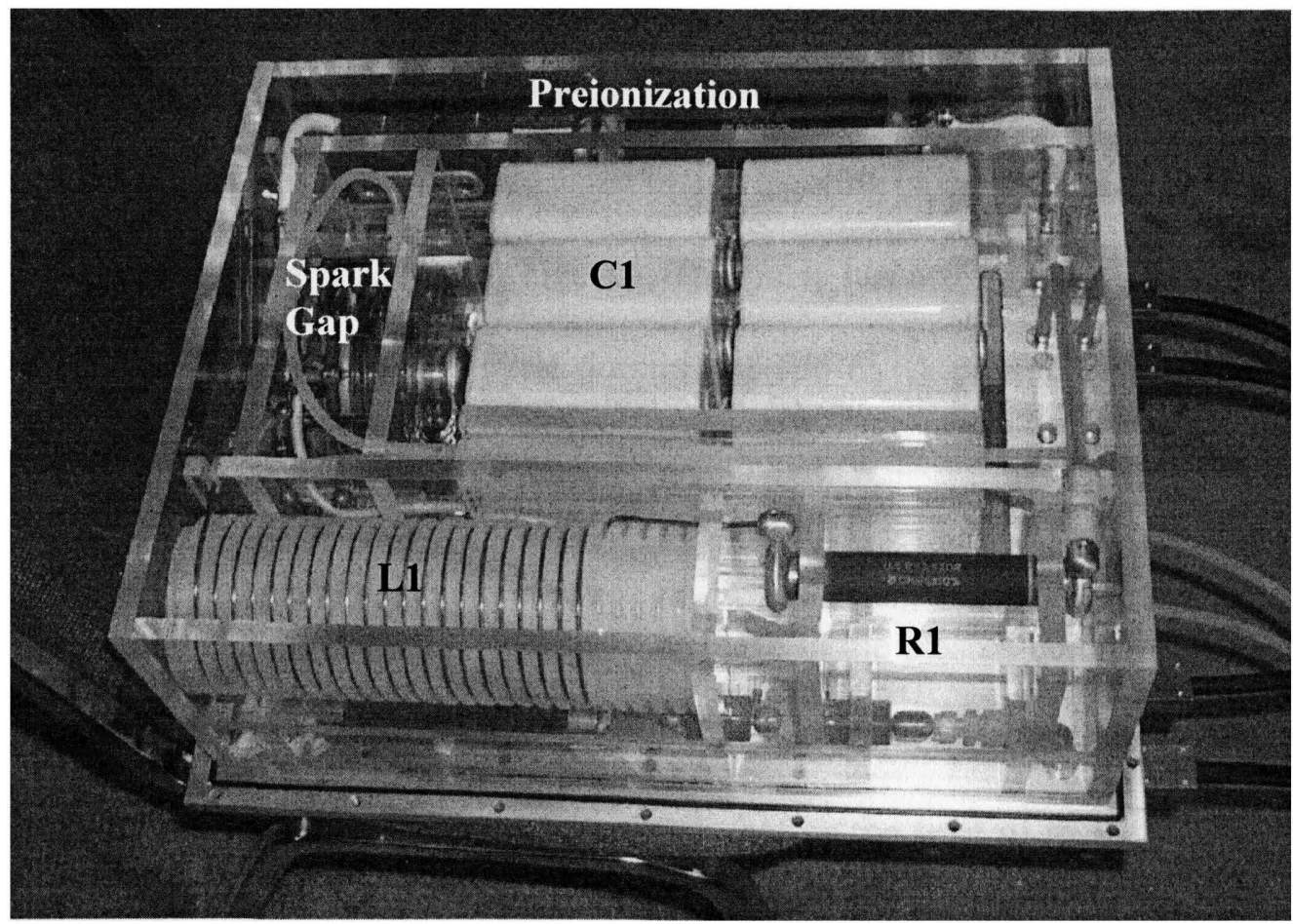

b
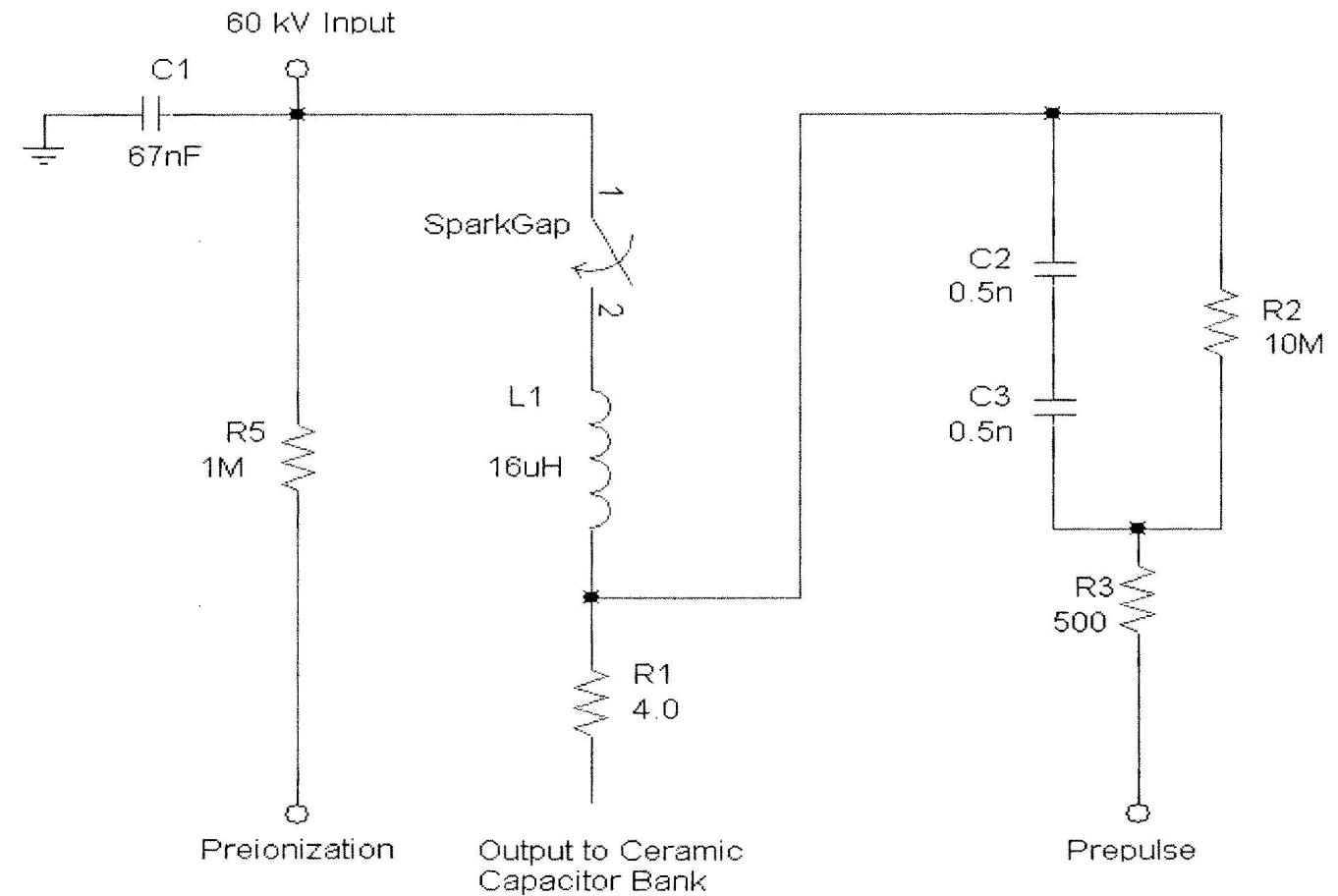

Prepulse

Figure 2.3: (a) Picture of the inside of new single stage pulse power unit (b) and schematic diagram. 
in figure 2.1a. This allows the synchronization of the laser output with external events with a jitter of several ns, as required in our specific application to photochemistry experiments. Sub-nanosecond jitter can also be obtained using laser triggering of the spark-gap [2.12].

A typical current pulse is shown in Fig. 2.4. The pulse has a $0 \%$ to $70 \%$ rise time of approximately $30 \mathrm{~ns}$, and a first half cycle duration of $165 \mathrm{~ns}$. A pronounced kink in the current is observed to occur about $38 \mathrm{~ns}$ after the beginning of the current pulse. This local minima of the current occurs at the time the plasma column reaches its minimum diameter of 200-300 $\mu \mathrm{m}$, and is caused by the significant increase in the plasma column inductance that accompanies the reduction of the plasma column diameter. The laser pulse of approximately $1.5 \mathrm{~ns}$ FWHM duration occurs shortly before the time of maximum plasma compression.

The capillary, the strontium titanate capacitors, and spark-gap are all contained in an $\mathrm{Al}$ enclosure that helps to shield the electromagnetic noise produced by the fast discharge. Biodegradable transformer oil is circulated for electrical insulation and also for cooling using a commercially available chiller unit. The laser light exits the cathode electrode that has a hole on axis and that is maintained at ground potential. Argon is continuously flown at the cathode end of the discharge, and is differentially pumped on one side of a $2 \mathrm{~mm}$ pinhole by a scroll pump and on the other side of the pinhole by a $360 \mathrm{l} / \mathrm{s}$ turbomolecular pump to avoid significant attenuation of the laser beam by photoionization of Ar atoms.

\subsection{Laser Output Pulse Energy and Timing Jitter Measurements}

The laser output pulse energy was measured using a vacuum photodiode placed at $80 \mathrm{~cm}$

from the exit of the laser and the data were recorded and stored by a $5 \mathrm{Gs} / \mathrm{s}$ digitizing oscilloscope. The quantum efficiency of the Al photocathode was previously calibrated with 


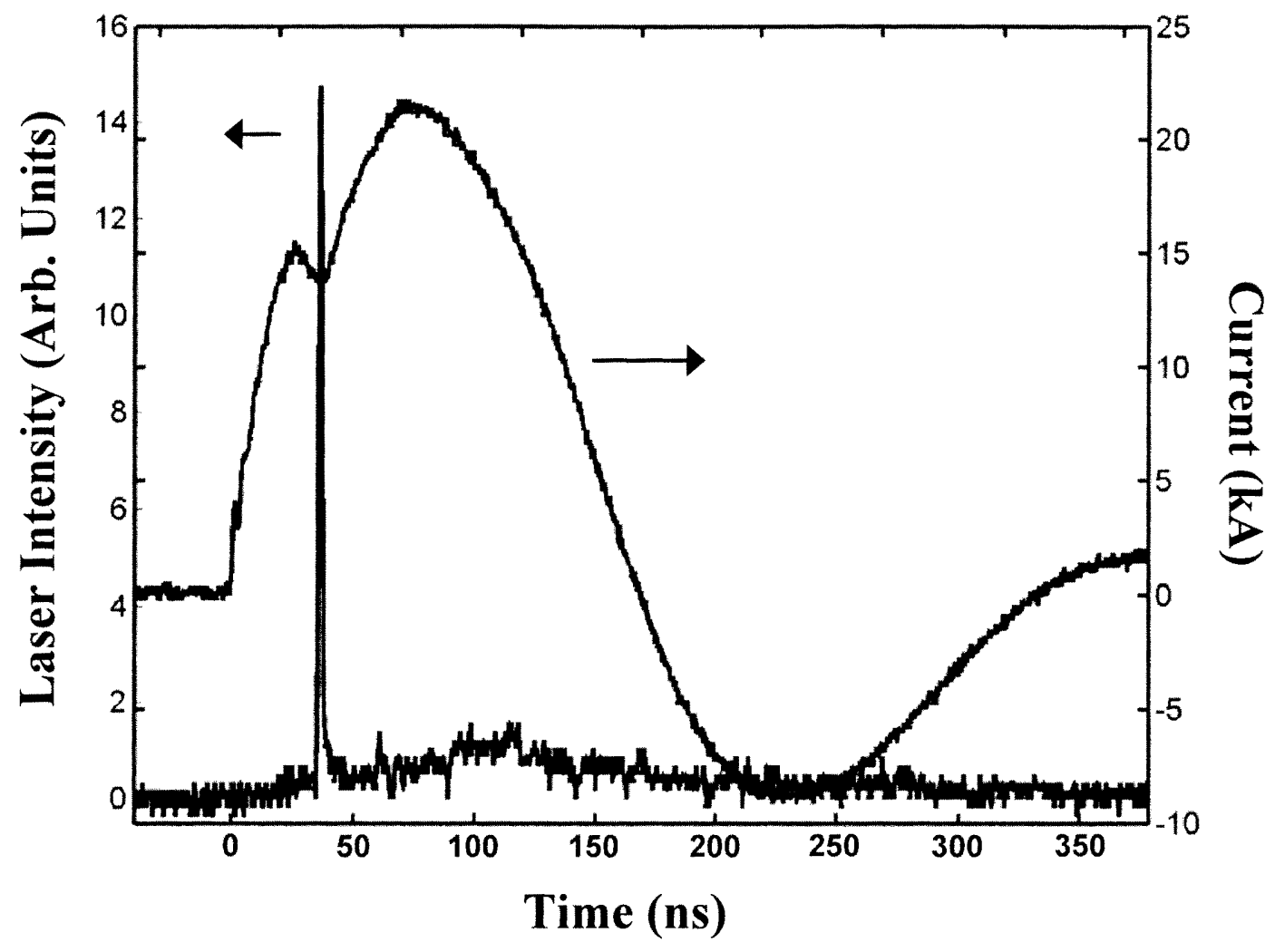

Figure 2.4. Discharge current pulse and laser output pulse. The kink in the current trace is caused by the abrupt increase of the plasma column inductance at the time of the pinch. 
respect to a silicon photodiode of known quantum yield [2.7]. The laser output was attenuated with several stainless steel meshes of measured transmissivity to avoid saturation of the photodiode. The laser was successfully operated at repetition rates up to $12 \mathrm{~Hz}$. Figure 2.5a shows the shot to shot variation of the peak of the excitation current pulse for 1500 consecutive shots at $12 \mathrm{~Hz}$ repetition rate. Figure $2.5 \mathrm{~b}$ and Fig $2.5 \mathrm{c}$ illustrate the corresponding shot to shot variation of the laser output pulse energy as a function of the shot number and its statistical distribution respectively. The average pulse energy is $13 \mathrm{uJ}$ and the standard deviation is $1.3 \mathrm{uJ}$, corresponding to an average power of about $0.15 \mathrm{~mW}$. The present experiments also demonstrated for the first time relatively low jitter operation of a capillary discharge soft $\mathrm{x}$-ray laser achieved by electrical triggering. Figure $2.5 \mathrm{~d}$ illustrates the statistical distribution of the time delay between the TTL trigger signal into the high voltage trigger unit that fires the spark-gap and the laser output pulse for the 1500 laser shots in Fig 2.5a. The standard deviation of the jitter is $5 \mathrm{~ns}$.

\subsection{Observations About the Capillary Lifetime}

Ablation of the capillary walls by the powerful discharge over a large number of shots increases their roughness, ultimately leading to the deterioration of the uniformity of the plasma column and to a consequent decrease of the laser output energy. Capillary lifetime tests were conducted at $12 \mathrm{~Hz}$ repetition rate recording the laser output energy for a large number of shots. The laser output pulse energy was measured to decay by a factor of 2 after about $210^{4}-310^{4}$ shots (Fig. 2.6). This is to our knowledge the longest series of soft x-ray laser shots achieved to date. The full output pulse energy can be recovered by replacing the used capillary discharge tube by a new one, an operation that demands 30 - 

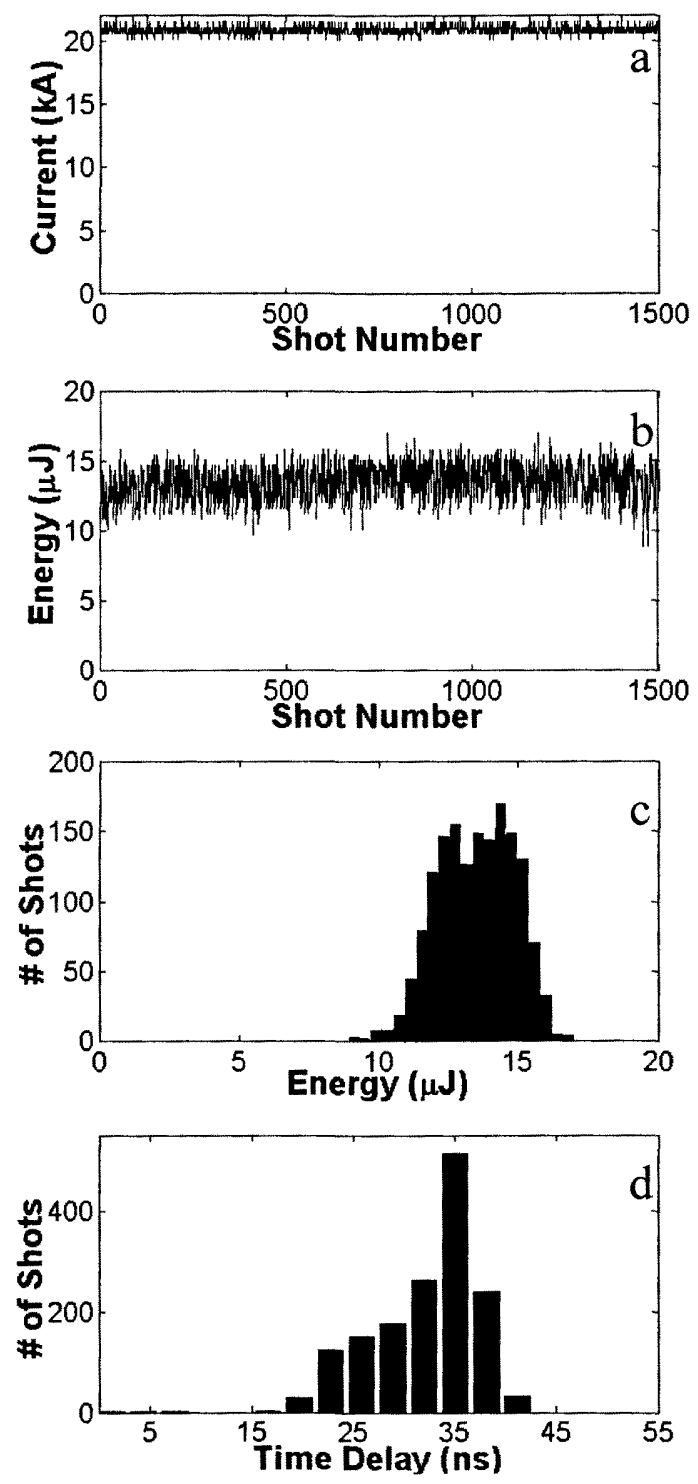

Figure 2.5. Data corresponding to 1500 shots of continuous $12 \mathrm{~Hz}$ repetition rate laser operation. (a) peak current; (b) measured laser output pulse energy; (c) distribution of the laser output pulse energy. The average pulse energy is $13 \mu \mathrm{J}$; (d) statistics of the time delay between the laser pulse and the TTL signal into the high voltage trigger unit. 


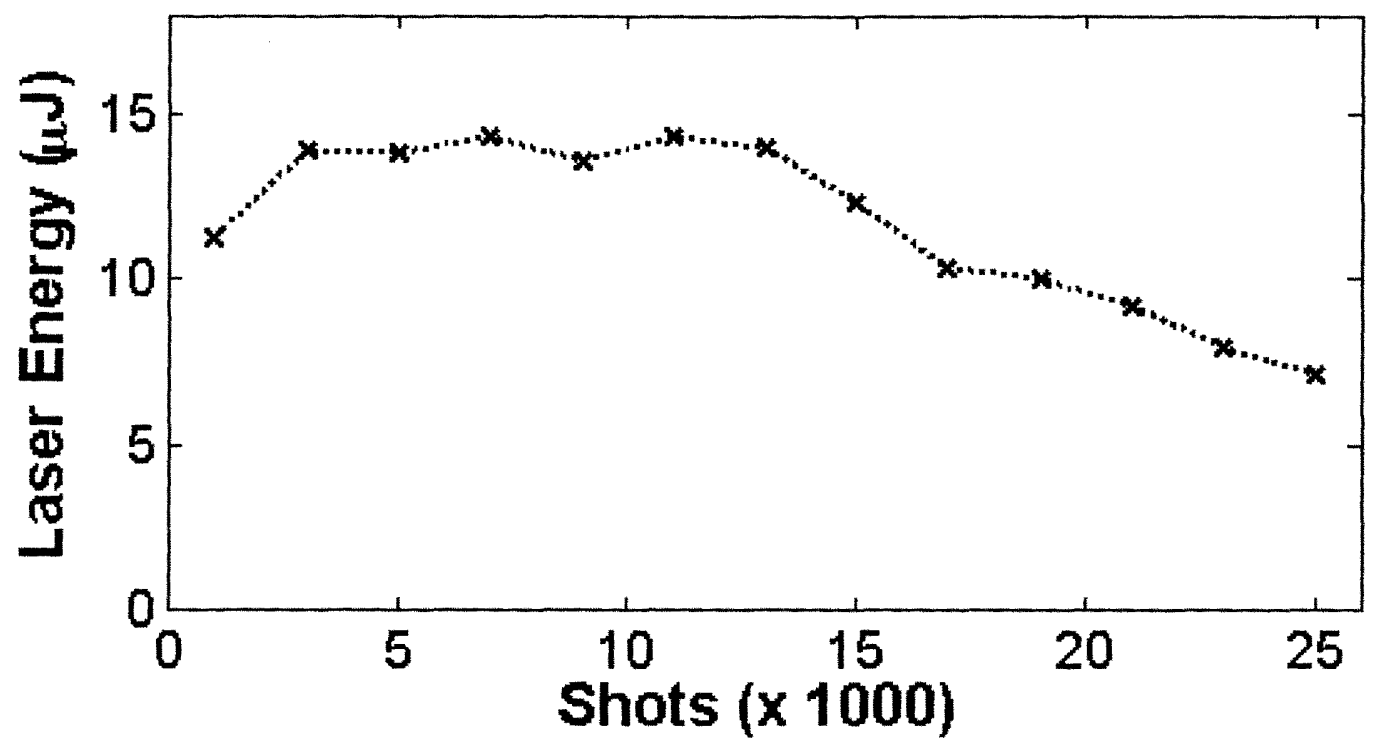

Figure 2.6. Variation of the laser output pulse energy as a function of the number of shots. The data was obtained operating the laser at $12 \mathrm{~Hz}$ repetition rate. The output energy is observed to degrade to half of the maximum value in about 2-3 $10^{4}$ discharge shots 
40 minutes including the pumping time required to evacuate the system to a pressure of $<$ $1 \times 10^{-5}$ Torr.

\subsection{Far Field Laser Output}

The far field laser output intensity distribution (Fig. 2.7a) was measured using a microchannelplate/phosphor screen read by a CCD array detector of 1024 X 1024 pixels placed at $157.5 \mathrm{~cm}$ from the exit of the laser. The microchannel plate was gated with a $5 \mathrm{~ns}$ voltage pulse to be able to discriminate the laser light from the long lasting spontaneous emission by the plasma in hundreds of extreme ultraviolet transitions. The beam profile was observed to have an annular shape that is the result of refraction of the amplified rays (as discussed in section 1.1) by radial electron density gradients in the plasma column [2.13-2.14]. Figure $2.7 \mathrm{~b}$ shows a cross section of an output intensity pattern acquired in a single shot. The peak-to-peak divergence is about $5.2 \mathrm{mrad}$.

\subsection{Summary}

In summary we have demonstrated high repetition rate $(12 \mathrm{~Hz})$ operation of a desktop $46.9 \mathrm{~nm}$ lasers that is easily transportable and has a relatively low timing jitter. To the authors knowledge this is the first demonstration of a soft $\mathrm{x}$-ray laser that can maintain an output energy of $>10 \mu \mathrm{J}$ at repetition rates up to $12 \mathrm{~Hz}$ with a total lifetime of $210^{4}-310^{4}$ shots and the first to be installed in a photochemistry lab. The laser is electrically triggered to allow synchronization with external events. This new type of portable short wavelength laser is of interest for numerous applications including experiments in photochemistry, materials characterization and patterning, and high resolution imaging.

The next chapter discusses the result of the use of this laser in nanocluster spectroscopy experiments. 

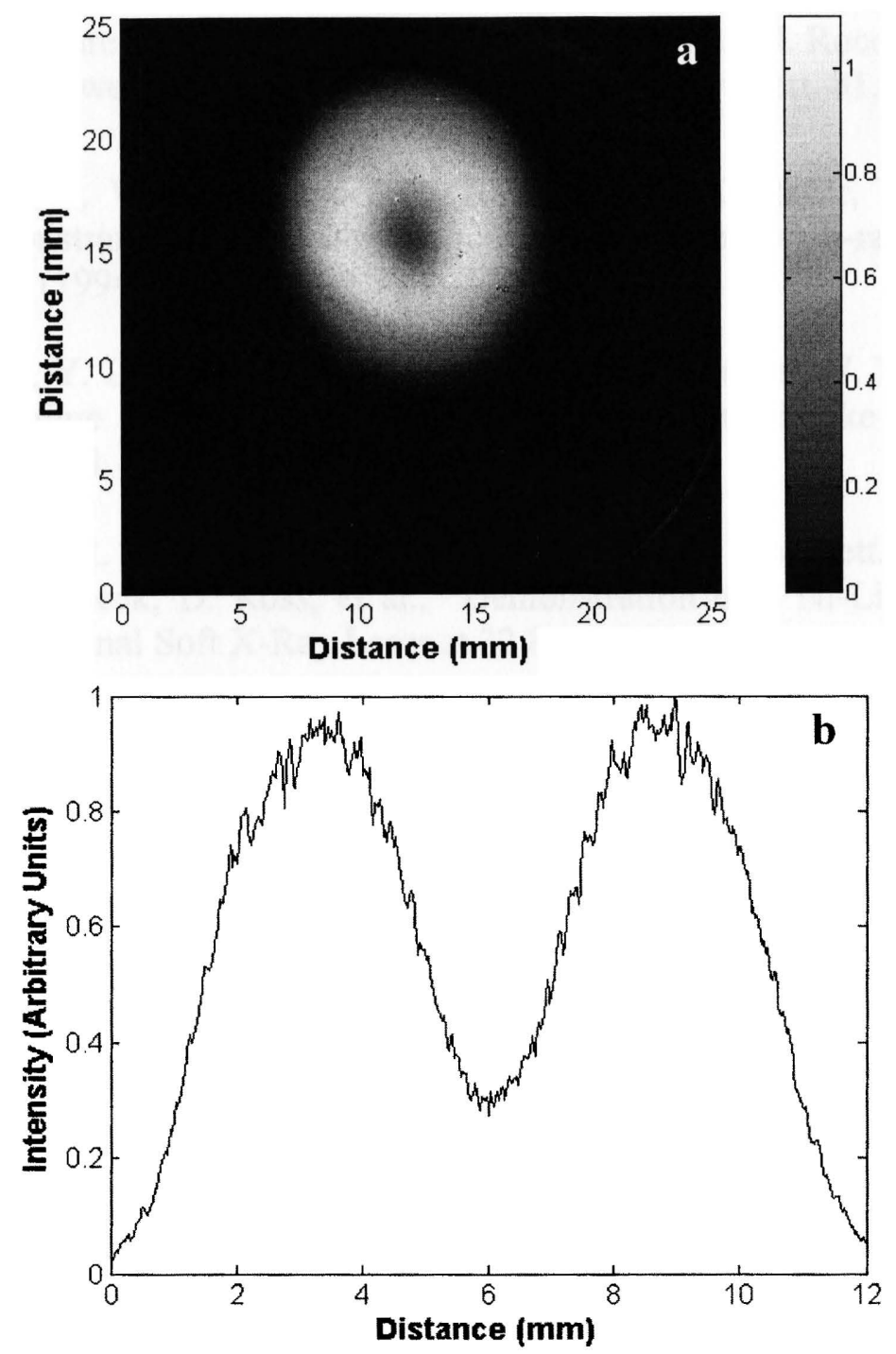

Figure 2.7. (a) Far field image of the laser beam measured at $157.5 \mathrm{~cm}$ from the capillary exit. (b) corresponding intensity lineout. The peak-to-peak beam divergence is $\sim 5 \mathrm{mrad}$. 


\section{References}

[2.1] B. R. Benware, C. D. Macchietto, C. H. Moreno, and J. J. Rocca, "Demonstration of a high average power table top soft x-ray laser," Phys. Rev. Lett. 81, 5804 (1998).

[2.2] J. J. Rocca, V. Shlyaptsev, F.G. Tomasel, O.D. Cortazar, D. Hartshorn, J.L.A. Chilla, "Demonstration of a discharge pumped table-top soft x-ray laser," Phys. Rev. Lett. 73, 2192, (1994).

[2.3] J. Dunn, Y. Li, A. L. Osterheld, J. Nilsen, J. R. Hunter, V. N. Shlyaptsev, "Gain Saturation Regime for Laser-Driven Tabletop, Transient Ni-Like Ion X-Ray Lasers," Phys. Rev. Lett. 84, 4834 (2000)

[2.4] S. Sebban,. R. Haroutunian, Ph. Balcou, et al., Phys. Rev. Lett. 86, 3004 (2001) and S. Sebban, T. Mocek, D. Ross, et al., "Demonstration of a Ni-Like Kr Optical-FieldIonization Collisional Soft X-Ray Laser at 32.8 nm," Phys. Rev. Lett. 89, 253901 (2002).

[2.5] K.A. Jenulewicz, A. Lucianetti, G. Pruebe, W. Sadner and P.V. Nickles, "Saturated Ni-like Ag X-ray laser at $13.9 \mathrm{~nm}$ pumped by a single picosecond laser pulse," Phys. Rev. A 68, 051802 (2003).

[2.6] A. Butler, A.J. Gonsalves, C.M. McKenna, D.J. Spence, S.M. Hooker, S. Sebban, T. Mocek, I. Betttaibi and B. Cros, "41.8-nm Xe ${ }^{8+}$ laser driven in a plasma waveguide," Phys. Rev. A 70, 023821 (2004).

[2.7] J. J. Rocca, D. P. Clark, J. L. A. Chilla, V. N. Shlyaptsev, "Energy Extraction and Achievement of the Saturation Limit in a Discharge-Pumped Table-Top Soft X-Ray Amplifier," Phys. Rev. Lett. 77, 1476 (1996).

[2.8] B. R. Benware, C. D. Macchietto, C. H. Moreno, and J. J. Rocca, "Demonstration of a high average power table top soft x-ray laser," Phys. Rev. Lett. 81, 5804 (1998).

[2.9] C. D. Macchietto, B. R. Benware, and J. J. Rocca, "Generation of millijoule-level soft-x-ray laser pulses at a $4-\mathrm{Hz}$ repetition rate in a highly saturated tabletop capillary discharge amplifier," Opt. Lett. 24, 1115 (1999).

[2.10] A. Ben-Kish, M. Shuker, R.A. Nemirowsky, A. Ron, and J.L. Schwob, "Plasma Dynamics in Capillary Discharge Soft X-Ray Lasers," Phys. Rev. Lett. 87, 1 (2001).

[2.11] A. Ritucci, G. Tomassetti, A. Reale, L. Palladino, L. Reale, F. Flora, L. Mezi, S.V. Kukhlevsky., A. Faenov, T. Pikuz, "Investigation of a highly saturated soft X-ray amplification in a capillary discharge plasma waveguide," Applied Phys. B 78, 965 (2004). 
[2.12] B. Luther, L. Furfaro, A. Klix, and J.J. Rocca, "Femtosecond laser triggering of a sub-100 picosecond jitter high-voltage spark gap," Appl. Phys. Lett. 79, 3248-3250 (2001).

[2.13] C.H. Moreno, M.C. Marconi, V.N. Shlyaptsev, B. Benware, C. Macchietto, J.L.A. Chilla, J.J. Rocca, "Two-dimensional near-field and far-field imaging of a Nelike Ar capillary discharge table-top soft-x-ray laser," Phys. Rev. A 58, 1509 (1998).

[2.14] J.L.A. Chilla and J.J. Rocca, "Beam optics of gain-guided soft-x-ray lasers in cylindrical plasmas," J. Opt. Soc. Am. B 13, 2841 (1996). 


\section{CHAPTER 3}

\section{FIRST RESULTS OF USE OF DESK-TOP $46.9 \mathrm{~nm}$ LASER IN TIME OF FLIGHT MASS SPECTROSCOPY NANOCLUSTERS EXPERIMENT}

The new type of portable laser described in the previous chapter is of interest for many applications that require a compact intense source of short wavelength laser light. One application that is currently being pursued is the study of metal oxide nanoclusters and other small molecules using time of flight mass spectroscopy (TOFMS). The project goal is to demonstrate a mass spectroscopy system for the study of the evolution of very dilute species such as gas phase clusters, based on single photon ionization made possible by a desktop EUV laser photoionization source.

\subsection{Motivation for studying metal oxide clusters}

Transition metal oxides are widely used as heterogeneous catalysts and catalytic supports in industrial processes. For example, the Monsanto process uses a vanadium oxide catalyst for catalytic oxidation of $\mathrm{SO}_{2}$ to $\mathrm{SO}_{3}$, which is an important step in production of sulfuric acid [3.1]. Also, titanium oxide catalysts have been found to photo-oxidize $\mathrm{CO}, \mathrm{C}_{3} \mathrm{~F}_{6}$, and $\mathrm{CH}_{3} \mathrm{Cl}$ to different sets of products depending on the oxide surface structure [3.1]. There are numerous other applications for transition metal oxide catalysts, and although they are widely used, there is a lack of fundamental understanding of the complicated processes that occur on the metal oxide surface during catalysis. Particularly, a complete understanding of the complex relationship between the structure of metal oxides, the reactivity of the metal oxide, and the processes by which these catalysts function is yet to be established. 
A metal oxide surface can be thought of as a collection of clusters of different sizes and isomers. The gas phase metal oxide clusters are regarded as the simplest model for the interaction of active sites on a transition metal oxide catalyst with organic molecules [3.2]. The effect of composition, stoichiometry, size, charge state, and oxidation state, which are among the most important factors affecting catalytic properties, can be examined by employing gas phase techniques as discussed by Castleman et al. in greater detail in reference 3.2 .

Also discussed by Castleman, the study of metal and metal compound clusters is serving to answer a wide variety of questions including size effects on reactivity, the nature and evolution of geometrical structure, and electronic properties toward the extended solid state of matter [3.3]. Predominantly relevant is determining how the molecules of a cluster evolve to a solid. It is well recognized that reactions on metal and metal compound clusters often display analogies to those which can occur on surfaces and therefore bear on an understanding of the physical basis for catalysis, etching phenomena, absorption phenomena, and many other observables. The importance of clusters can be summarized as: 1) clusters provide a theoretical and experimental bridge between the molecular and nanostructure regimes; 2) clusters provide a path to the explanation of catalytic activity under isolated, controlled, and reproducible conditions: and 3) clusters provide a detailed mechanistic model for condensed phase and surface catalytic reactivity and reactions. A more extensive discussion on the understanding of nanoclusters can be found in references $[3.1-3.3]$.

\subsection{Why an EUV Laser Source?}

Various spectroscopy studies have been conducted for nanoclusters of many different 
types and sizes [3.1-3.8]. Summarizing section 3.1, these experiments are used to gain information about the reactivity and catalytic behavior of nanoclusters and how these properties change during the evolution of a nanocluster molecule into a solid. For the exploration of neutral cluster properties and distributions, one must first ionize the gas-phase clusters in order to use mass spectroscopy as a detection technique. For example, conventional nanocluster spectroscopy techniques have used $193 \mathrm{~nm}$ radiation from an ArF excimer laser corresponding to a photon energy of $6.4 \mathrm{eV}$ in order to photoionize a sample $[3.1,3.4,3.8]$. Typical hydrogen bonded and metal oxide nanocluster ionization energies fall into the range of 7-12 eV while some have even higher energies (see table 3.1). Therefore a single $6.4 \mathrm{eV}$ photon can not ionize the cluster making multiphoton processes the dominant ionization method (see figure 3.1). During the multiphoton ionization process, at least two progressions can be responsible for cluster ionization: (1) a cluster can absorb a photon through low-lying electronic states and relax back to the ground electronic state many times, thus heating the cluster until thermionic emission occurs, and (2) absorption of two or more photons can occur without rapid relaxation between absorption steps for both neutral and/or ionic species [3.4].

A major problem associated with mass spectroscopy can become evident during the multiphoton ionization of clusters. Specifically, the clusters may fragment during the ionization process and the identification of the neutral parent cluster can become difficult. This problem can be reduced by using radiation with more energy per photon that can ionize these systems with a single photon. One wavelength that has been explored is $118 \mathrm{~nm}$ (10.5 $\mathrm{eV} /$ photon) from the ninth harmonic of Nd:YAG laser light that can ionize many clusters using a single photon and significantly reduce negative fragmentation effects [3.1, 3.4]. 
Table 3.1: Ionization energy values for some common small metal oxide and hydrogen bonded clusters.

\begin{tabular}{c|c} 
Metal Oxide Cluster & Ionization Energy (eV) \\
\hline $\mathrm{FeO}$ & 8.5 \\
$\mathrm{FeO}_{2}$ & 9.5 \\
$\mathrm{TiO}$ & 6.8 \\
$\mathrm{TiO}_{2}$ & 10 \\
$\mathrm{VO}$ & 7.5 \\
$\mathrm{VO}$ & 10.3 \\
$\mathrm{ZrO}_{2}$ & 6.2 \\
$\mathrm{ZrO}_{2}$ & 9.4 \\
$\mathrm{H}_{2} \mathrm{O}$ & 12.6 \\
$\mathrm{NH}_{3}$ & 10.1 \\
$\mathrm{CH}_{3} \mathrm{OH}$ & 10.8
\end{tabular}




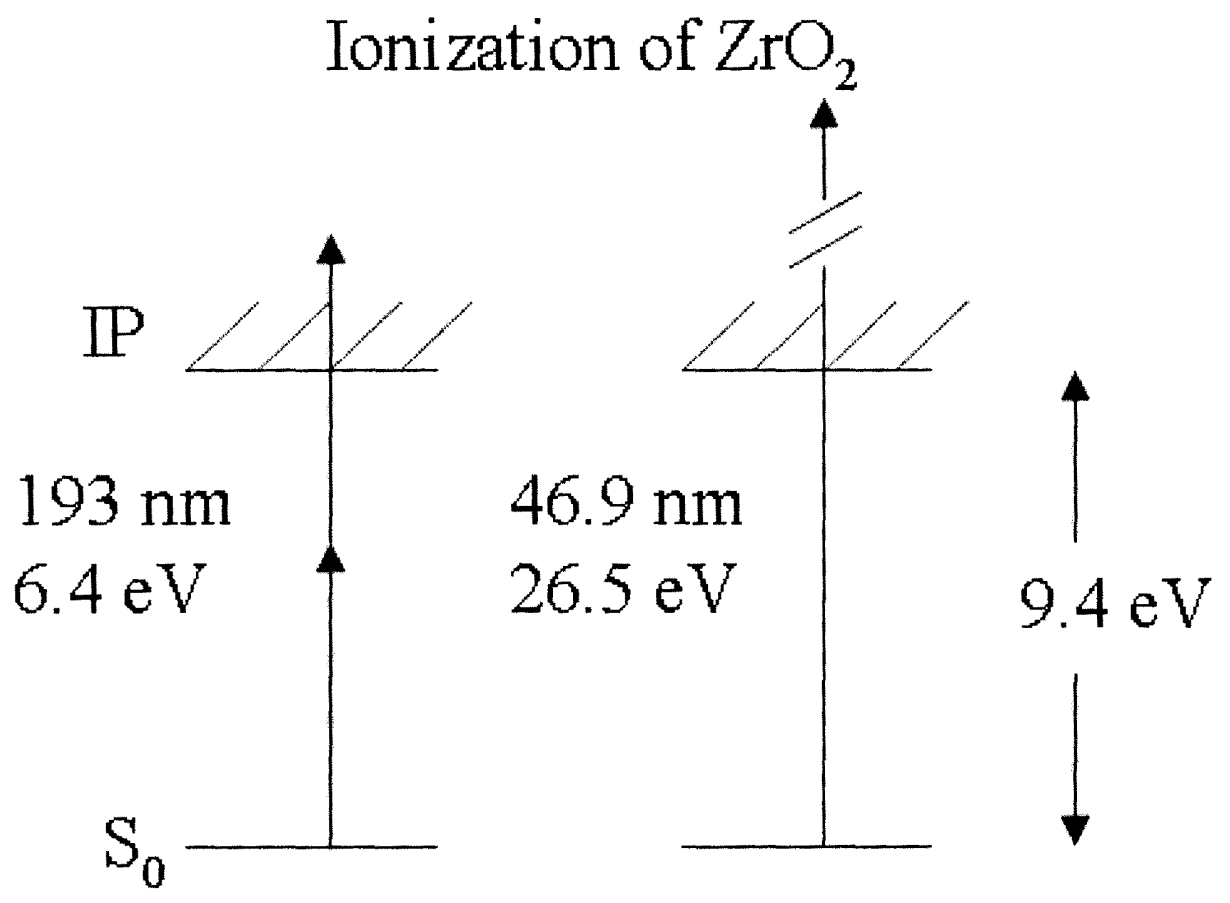

Fig. 3.1. Diagram depicting multiphoton ionization vs. single photonionization of $\mathrm{ZrO}_{2}$ metal oxide nanoclusters. 
However, $118 \mathrm{~nm}$ can not access all cluster series with a single photon. This thesis presents the first desk-top size soft $\mathrm{x}$-ray laser to be used as a photoionization source in TOFMS experiments to study nanocluster systems that can ionize any small molecule with a single photon of light.

The EUV laser source should drastically change the ionization process so that multiphoton effects are greatly reduced. A single photon of the EUV laser provides $26.5 \mathrm{eV}$ of energy, which is more than enough energy to ionize clusters that typically have ionization energies in the range of 7-12 eV with a single photon, thus reducing loss of parent cluster mass information. Also, the high energy per photon of the source allows us to ionize any gas or product of a reaction with a cluster including $\mathrm{O}_{2}, \mathrm{NO}, \mathrm{NO}_{2}, \mathrm{~N}_{2}, \mathrm{CO}, \mathrm{CO}_{2}$, and many others. This is a valuable asset when studying the reactivity of the clusters. In addition, the TOFMS system constitutes a method of testing the laser and its application to science in which it has to operate in a typical chemistry laboratory environment for many hours at a time in combination and synchronization with a Nd:YAG laser and other instrumentation.

\subsection{Nanocluster Spectroscopy Apparatus Based on 46.9 nm Laser}

This section discusses a mass spectroscopy apparatus that is used to obtain data involving nanoclusters and other small molecules and is based on the $46.9 \mathrm{~nm}$ laser described in Chapter 2. The apparatus and procedures are very similar to experiments done previously $[3.1,3.4,3.8]$ with the exception of the soft $\mathrm{x}$-ray laser as the photoionization source. A schematic representation of the experiment can be seen in figure 3.2.

Neutral metal oxide clusters are produced in a conventional laser vaporization/supersonic expansion cluster source by laser ablation of a metal foil or metal 
drum into helium carrier gas mixed with up to $10 \%$ of $\mathrm{O}_{2}$. The pulsed oxygen/helium flow from an R.M. Jordan Co. pulsed nozzle [3.9] operating at a backing pressure of 80 psi is injected through 0.8 -mm-diam nozzle hole into an $\sim 6$-cm-long flow channel in the laser ablation source. The metal of interest is ablated at laser energies of $\sim 20 \mathrm{~mJ} / \mathrm{pulse}$ by a 532 $\mathrm{nm}$ wavelength laser pulse from the second harmonic of a $\mathrm{Nd} / \mathrm{YAG}$ laser directed perpendicularly to the carrier gas channel and focused by an $f=75 \mathrm{~cm}$ lens on the surface of a rotating and translating drum wrapped with a metal foil. The high energy per pulse $(\sim 10$ $\mu \mathrm{J})$ and repetition rate of several $\mathrm{Hz}$ of the capillary discharge $46.9 \mathrm{~nm}$ laser developed for this experiment is well matched with the repetition rate of the commercial Nd-YAG laser for sample ablation. The metal foil surface is tangential to the ablation channel wall and the ablation spot is approximately $2.75 \mathrm{~cm}$ downstream from the nozzle orifice. Another 3.25 $\mathrm{cm}$ downstream from the ablation spot, the clusters entrained in the carrier gas flow freely, expand into vacuum, and are carried by the ensuing molecular beam through a $1.5 \mathrm{~mm}$ skimmer into the ion source of a time of flight mass spectrometer (TOFMS) where they are ionized by the EUV $46.9 \mathrm{~nm}$ laser beam.

The EUV laser pulse energy is $\sim 10 \mu \mathrm{J}$ at the output of the laser, but is reduced to $\sim 1$ $\mu \mathrm{J}$ at the output of a $\mathrm{z}$-fold mirror system placed just before the ionization region with the purpose of providing alignment capability of the beam with respect to the nanocluster setup. The z-fold consists of two, 1 in. diameter $\mathrm{Sc} / \mathrm{Si}$ multilayer mirrors with about $40 \%$ reflectivity each [3.10]; a planar mirror placed $30.5 \mathrm{~cm}$ from a $f=50 \mathrm{~cm}$ concave mirror that focuses the incoming beam into the ionization region. The use of optics improves 


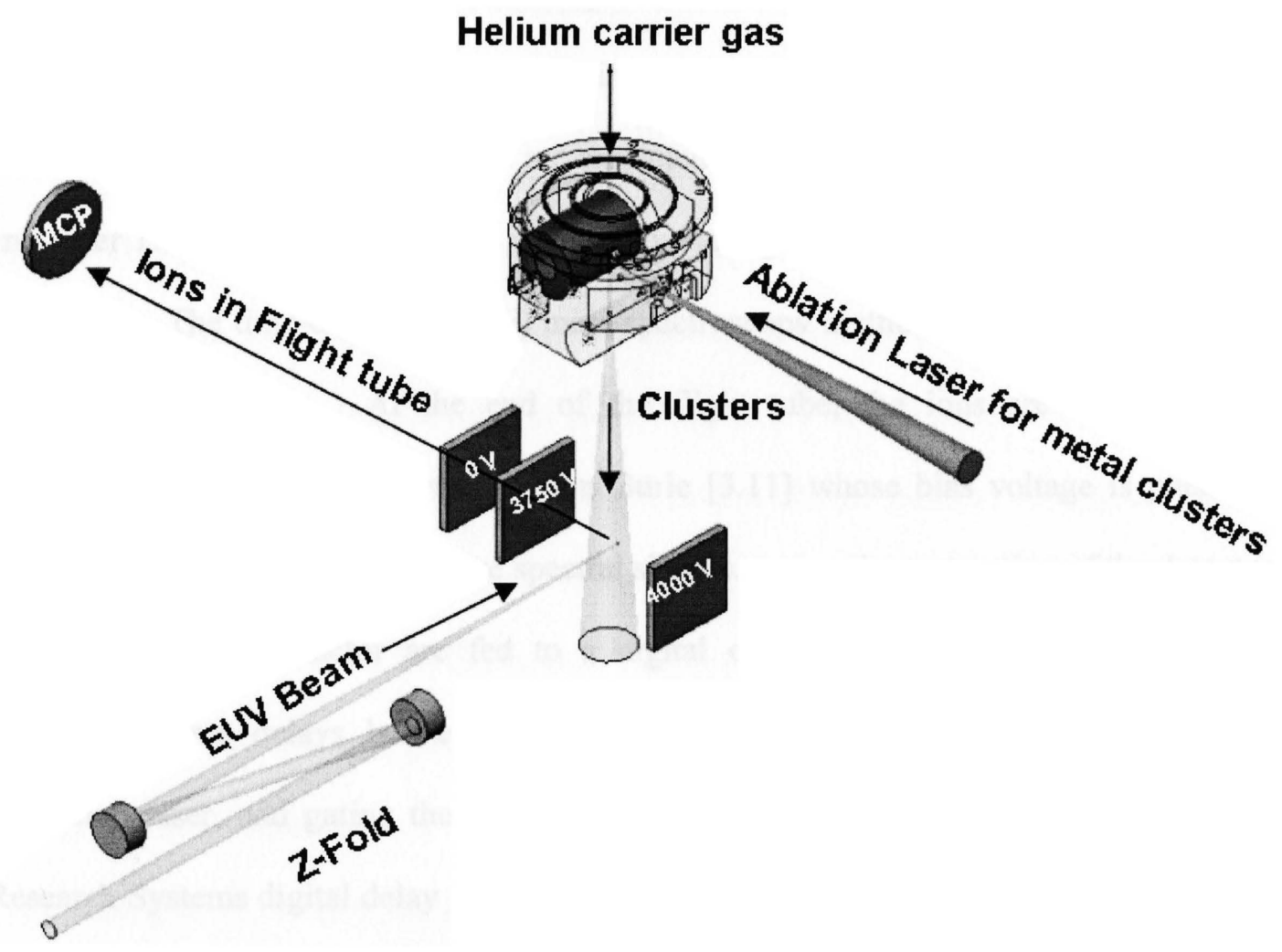

Fig. 3.2. Schematic representation of the nanocluster experiment. Metal oxide clusters are generated through laser ablation of the metal and reaction with various concentrations of $\mathrm{O} 2$ in He. A gas jet carries the metal oxide clusters to the ionization region. Clusters are ionized by the EUV laser photons. The cluster ions are analyzed by a TOFMS. 
the experimental setup in three ways: 1) two mirrors allow full directional control of the ionization beam in order to improve alignment; 2) the ability to scan the ionization region for overall improvement of signal intensity; and 3) the improved intensity of the beam using a focusing mirror for a tight focal spot in the interaction region. The focal spot has not been measured; however, it can be estimated assuming a Gaussian beam profile to be $\sim 10 \mu \mathrm{m}$. The cluster ions produced are extracted perpendicularly to the molecular beam and enter $1.0-\mathrm{m}$-long flight tube in which they are separated in arrival time according to their mass. The time-of-flight (TOF) mass spectroscopy method is discussed in detail in the following section. At the end of the flight tube, the ions are detected by a microchannel plate (MCP) detector from Burle [3.11] whose bias voltage is pulsed in order to avoid the detection of large spectral signals, preventing saturation of the detector. Signals from the detector are fed to a digital oscilloscope through a $50 \Omega \mathrm{MHV}$ connector. Time delays between valve opening, firing the ablation laser, firing the ionization laser, and gating the MCP's are generated by three programmable Stanford Research Systems digital delay generators DG 535 [3.12]. All timings can be adjusted in order to maximize the spectral signal strength.

\subsubsection{Time-of-flight mass spectroscopy (TOFMS)}

Positive ions are created in the ionization region of the mass spectrometer by exposing the clusters or small gas molecules to photons from the EUV laser, causing an electron to be ejected. An ionizing photon pulse of $\sim 1.5$ nanoseconds duration from the $46.9 \mathrm{~nm}$ EUV laser yields ion packets that are at least 1.5 nanoseconds wide. This defines the temporal distribution of the ion packet. The ion packet width is also 
determined by the position of the ions when first created or accelerated. This variation in position is known as the initial spatial distribution.

\subsubsection{The Ion Source}

The ions are created in the ionization region that is defined by the three plates illustrated in figure 3.3; the repeller plate, the extraction grid, and the accelerating grid. The spacing, $d$, between the plates must be kept small compared to the width, w, of the plates. This prevents penetration by outside electric fields which would distort the shape of the ionization and extraction fields. The ratio $\mathrm{w} / \mathrm{d}$ is usually three or four to one. The ions are produced between the repeller plate, which is held at a positive voltage to repel positive ions, and the extraction grid, which is held at a lower positive voltage creating an electric field that will accelerate the ions towards the flight tube. The space between the two plates is referred to as the ion source. The ions pass through the extraction grid and are accelerated by the third plate, the accelerating grid, which is typically held at zero voltage for two reasons. First, it is a lower voltage than both the repeller plate and the extraction grid creating a field that will accelerate a positive ion perpendicular to the plates and into the flight tube. Second, it acts as a boundary into the flight tube that is also held at zero potential, creating a field-free region. The flight tube will be discussed in more detail later in the section. Typical voltages for the three plates are shown in figure 3.3a.

At this time, the discussion will be simplified by considering the evolution of an ion packet modeled by three ions created in the ion source shown in figure 3.3. Figure 3.3a shows an ion close to the extraction grid that leaves the ion source first (3.3b) but it 


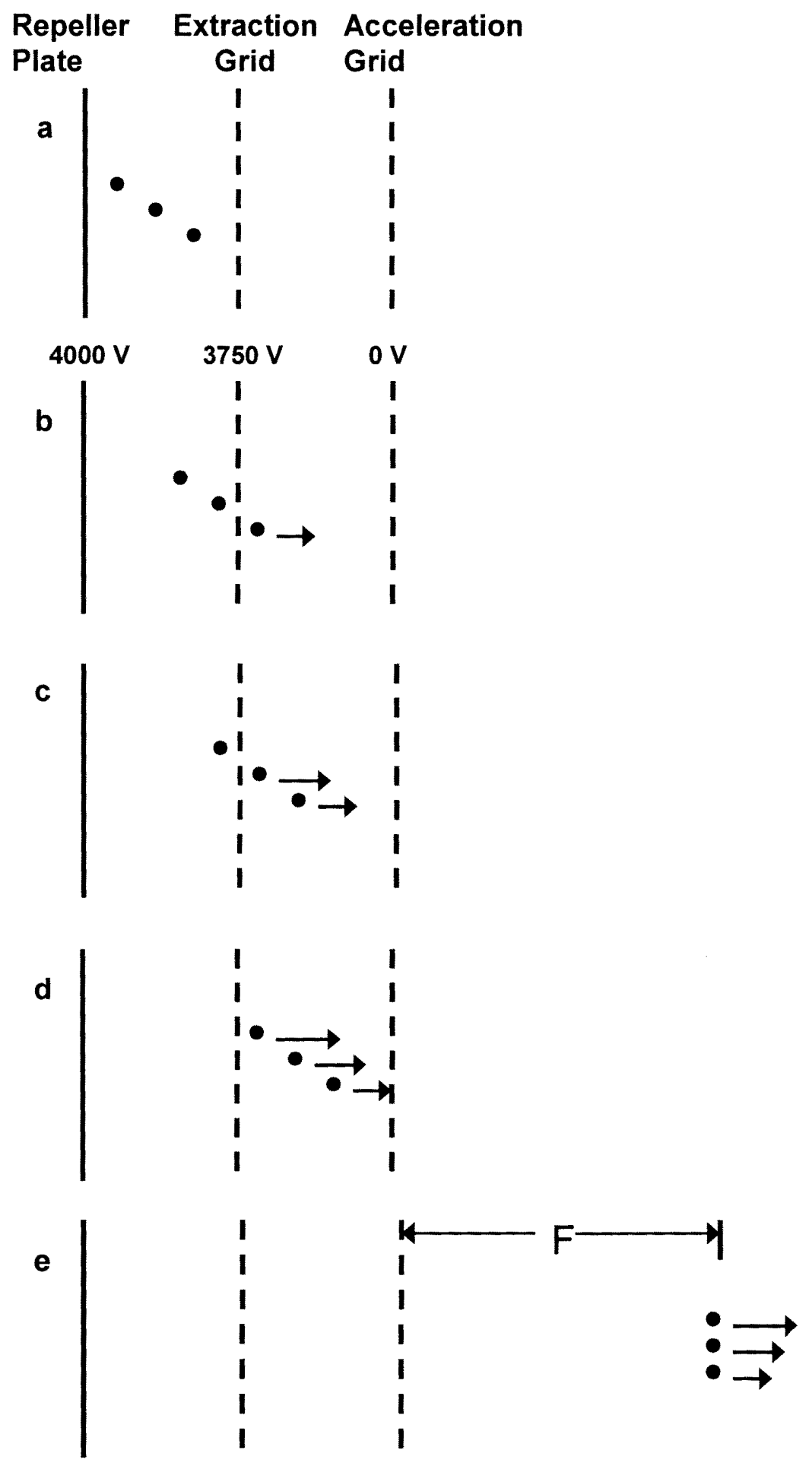

Figure 3.3: Schematic representation of the ionization region of a TOFMS (a) the creation of an ion packet between the repeller plate and extraction grid (b) ion closest to extraction grid leaves first but moves slowest (c) middle ion leaves second and faster (d) farthest ion leaves last and moving faster still (e) all catch each other at primary focal point in the flight tube. This figure is modified from figures in reference 3.9. 
moves slowly because it is exposed to the accelerating field for a small amount of time. The middle ion leaves later (3.3c), and will be moving faster, and the furthest ion leaves last (3.3d) and will be moving even faster. This variation in their velocities is referred to as the energy distribution. After traveling some distance, the late, faster ions catch up with the slower ones. This point is known as the primary focal point (3.3e). By adjusting the grid voltages and spacing one can control the location of this point called energy focusing. If the detector is located at point $\mathrm{F}$, the resolution is best, meaning that the width of the ion packet in time would be at its narrowest point.

Until now we have been considering molecules, which are static when ionized. An ion source must also deal with another kind of energy, called transverse energy arising from the component of kinetic energy of the ions in the plane of the electrodes. If an ion is moving at the time of its extraction pulse, its initial velocity can cause it to drift away from the axis of the flight tube. To counteract this, steering plates are added directly following the accelerating grid ensuring an on axis entrance into the flight tube shown in figure 3.4. A positive voltage on a plate, which generates to a force that is opposite in direction to the transverse velocity, will repel a positive ion back onto a desired course. Likewise, a negative voltage on the other plate will have the same effect. It is best to apply both potentials at the same time to keep the field symmetric. After exiting the ion source and acceleration region, the ions enter the flight tube.

\subsubsection{The Flight Tube}

The flight tube is usually a vacuum enclosure, free of electromagnetic fields, between the ion source and the detector. It is sometimes referred to as a field free drift region. The 


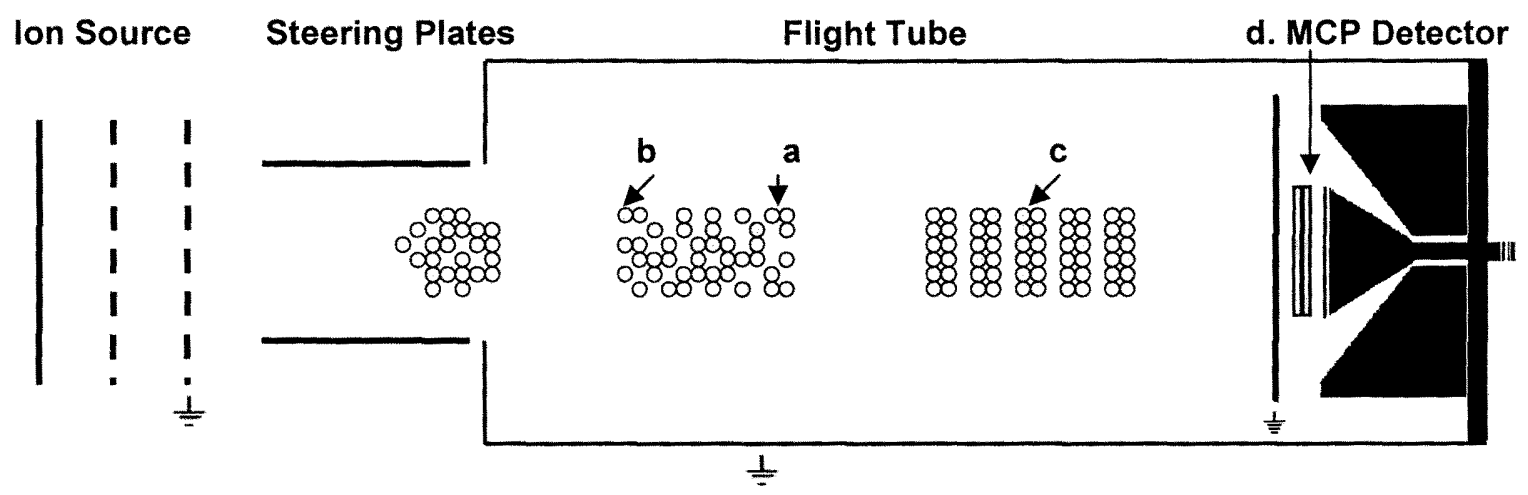

Figure 3.4. Time of flight mass spectrometer schematic that includes the ion source, steering plates, flight tube, and MCP detector. (a) Lighter ions travel ahead of (b) heavier ions until they are separated into (c) distinct ion packets separated by mass number. They are detected by an (d) MCP detector located at the end of the flight tube. This figure is modified from figures in reference 3.9 . 
ions exit the ion source and enter the flight tube with the proper velocity and direction to arrive at the detector. The flight tube does not interact with the ion packets along their flight path. The ions are created at a positive voltage and accelerated to ground potential, so the flight tube can be a simple pipe. Adequate pumping is required to prevent the ions from colliding with molecules on the way to the detector. The detector which is a microchannel plate must be operated at pressures below $1 \times 10^{-5}$ torr. This usually determines the maximum allowable pressure in the flight tube.

Ions enter the flight tube in packets that are defined by the EUV laser pulse width. All ions are given the same energy in the source. Since the same force is applied to the light ions as the heavy ions, the light ones will travel faster. Since they are traveling at different velocities, the ion packets become separated as they travel down the flight tube. The light ions will travel in front (3.4a) and the heavier ones in back (3.4b). Given sufficient flight time, the ions become separated into individual packets by mass number (3.4c). The detector amplifies them sequentially, and each packet becomes a mass peak with a kinetic energy given by $\mathrm{KE}=1 / 2 \mathrm{mv}^{2}$ where $\mathrm{v}$ is the velocity of the ion and $\mathrm{m}$ is the corresponding mass of the ion.

\subsubsection{The Detector}

A microchannel plate (MCP) detector is located at the end of the flight tube. At the end of the flight tube the ions reach the entry grid to the detector shown as figure $3.5 \mathrm{a}$. The grid is kept at the same voltage as the flight tube. It serves two functions similar to the accelerating grid in the ion source. The first is to act as an exact end in space for the 


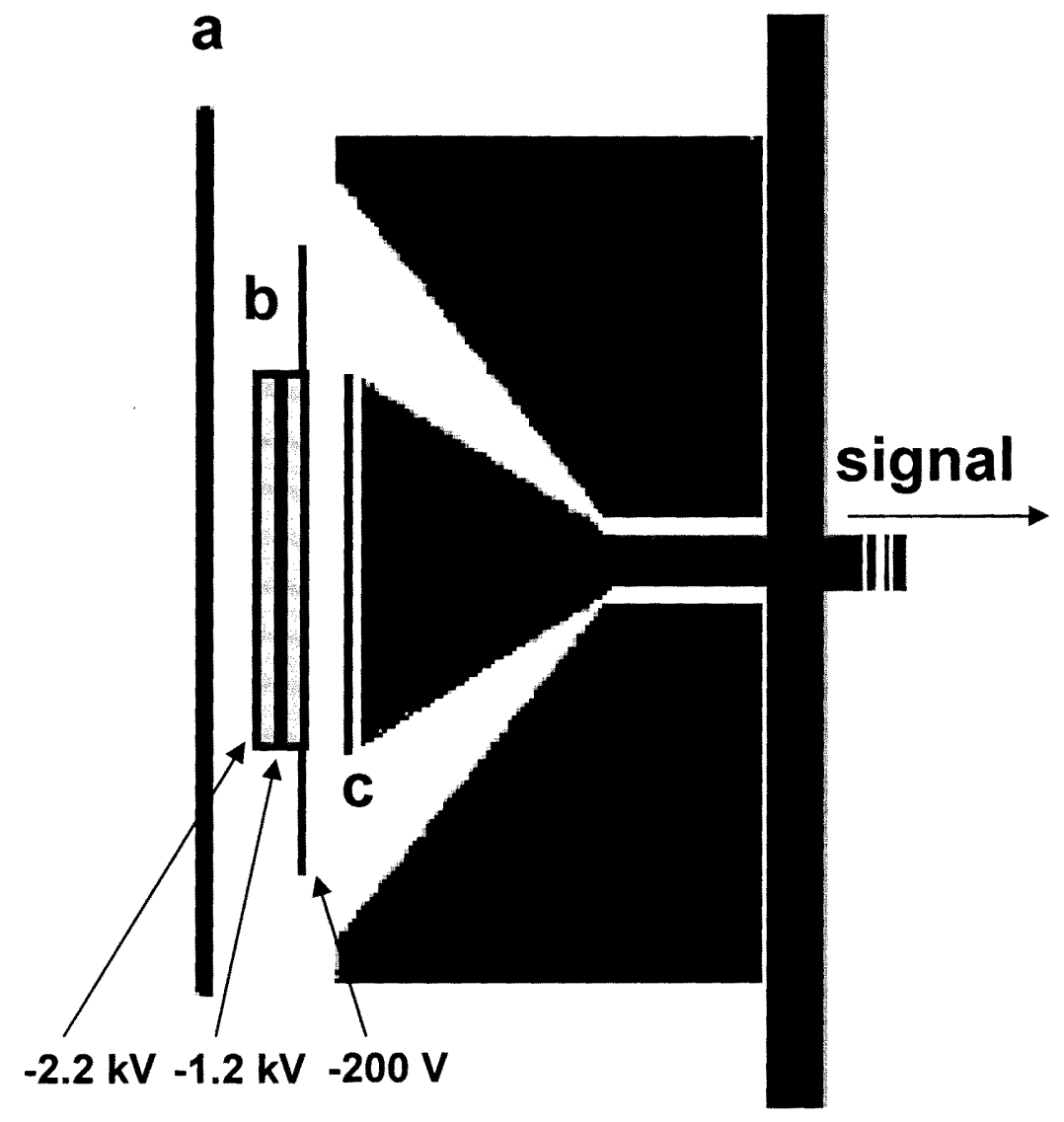

Figure 3.5: (a) The entry grid to the MCP detector is held at the same potential as the flight tube to ensure a field-free region and acts as a finish line for the ions. (b) the microchannel plates create and avalanche of electrons, initiated by the ion colliding with the top plate, that are collected by the (c) anode. This figure is modified from figures in reference 3.9 . 
drift region. This is the finish line for the ions in their trip down the flight tube. The second function of the entry grid is to shield the flight tube from the high voltages that are used in the detector. This insures a field free drift region all the way to the end of the flight tube. Immediately after passing through the grid, the ions are accelerated to a collision with the top of the first microchannel plate, which is held at a $-2.2 \mathrm{kV}$ potential seen in figure $3.5 \mathrm{~b}$. This collision jars loose one or more electrons from a channel of the plate. They, in turn go bouncing down the channel. At each collision, additional electrons are freed. These secondary electrons in turn bounce down the channel, creating still more electrons. This avalanche continues all the way through the plates so that over a million electrons come out of the bottom of the plates for each ion that hits the front (MCP gain is $\sim 10^{7}$, that is, 1 ion gives $10^{7}$ electons). These electrons are collected by a clean, flat plate shown as figure $3.5 \mathrm{c}$. The potential on the plate is more positive than the electrons so it is called the anode. The signal is now an electrical current passing through a solid metal conductor. The signal is fed to a digital oscilloscope that displays the mass spectrum of the sample under study. If the reader would like more details on TOFMS, please see reference 3.9.

\subsection{Results}

This section presents the first data obtained in the using the EUV laser-based mass spectroscopy setup. The following results are based on data taken in the study of small gas molecules, hydrogen bonded cluster systems, and preliminary efforts to obtain metal oxide cluster systems. A soft $\mathrm{x}$-ray laser has never before been used in photochemistry experiments so the first important observation to be made is that we can obtain useful, 
a)

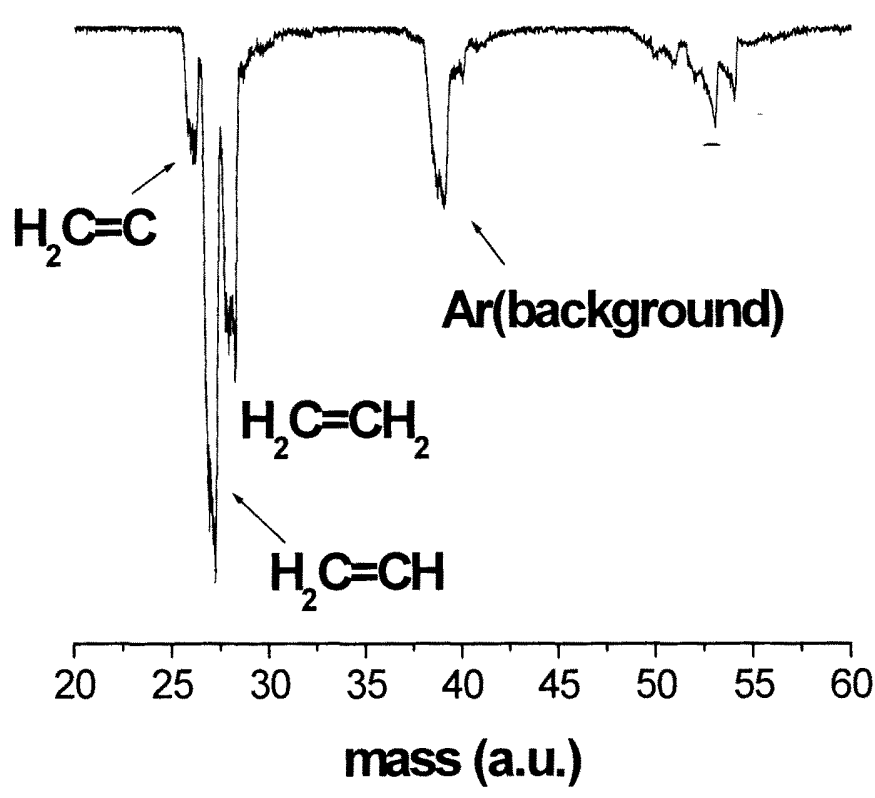

b)

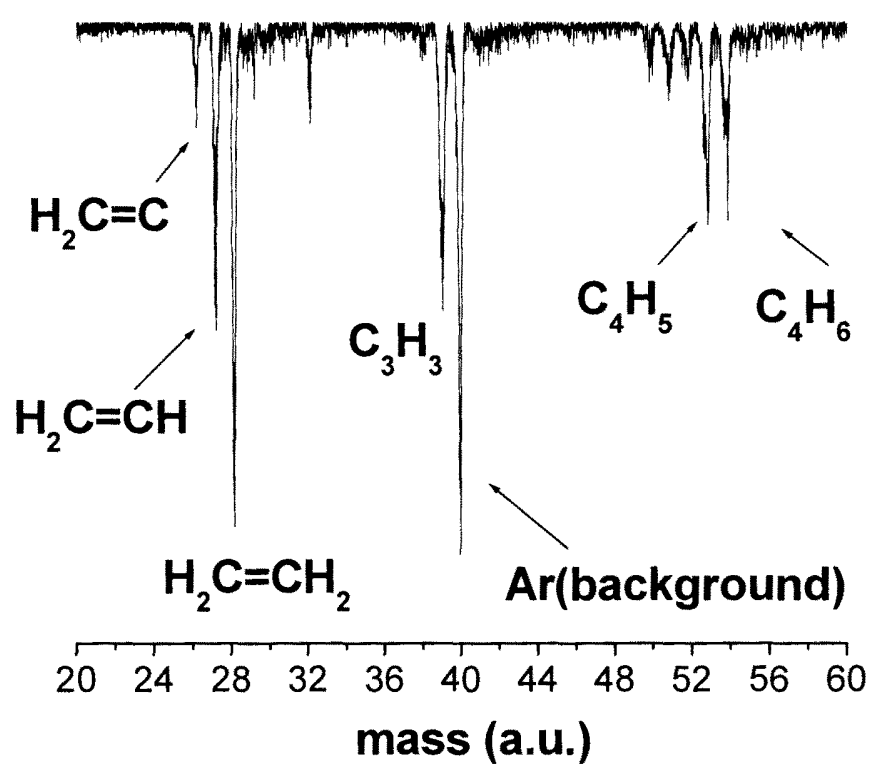

Figure 3.6: Comparison of mass spectra with a Methylene sample. a) With ASE included in the signal the resolution is about 130 ns FWHM and b) without ASE the resolution improves to about 10 ns FWHM. 
presentable data. Figure 3.6 presents the time of flight (TOF) mass spectrum of methylene generated and detected employing $46.9 \mathrm{~nm}$ single photon ionization. Fig. 3.6a represents data that includes amplified spontaneous emission (ASE) crated by the Ar plasma of the laser source. This produces poor spectral resolution where mass peaks are indistinguishable, making data analysis impractical. With the z-fold mirror system discussed earlier in place (see figure 3.2), the ASE is effectively reduced and the mass spectrum seen in figure $3.6 \mathrm{~b}$ is now produced. Mass peaks separated by one mass unit with good spectral resolution are observed. The FWHM of the peaks is approximately 10 ns.

Figure 3.7 compares data taken with and without pulsing the bias voltage on the MCP detector using ammonia $\left(\mathrm{NH}_{3}\right)$ seeded in helium $(\mathrm{He})$ carrier gas as a sample. If voltage is supplied to the MCP's at a time before the He signal (figure 3.7a), the spectrum shows a large He signal and weak signal coming from the ammonia sample. However, if the voltage is supplied at a time after the He ions arrive at the detector shown in figure $3.7 \mathrm{~b}$, the He signal is systematically omitted from the spectrum and intensity in the $\mathrm{NH}_{3}$ signal is gained telling us that the MCP is being saturated. Pulsing the bias voltage effectively gets rid of large unwanted signals in a spectrum and allows the MCP detector to operate in an unsaturated regime.

Complementary to a spectrally resolved and unsaturated data signal seen in the small molecules of ammonia and methylene, the soft x-ray laser can show us cluster series of hydrogen bonded systems that can not be accessed by a single photon VUV light. The hydrogen bonded cluster systems were originally included in this study because of the 
a)

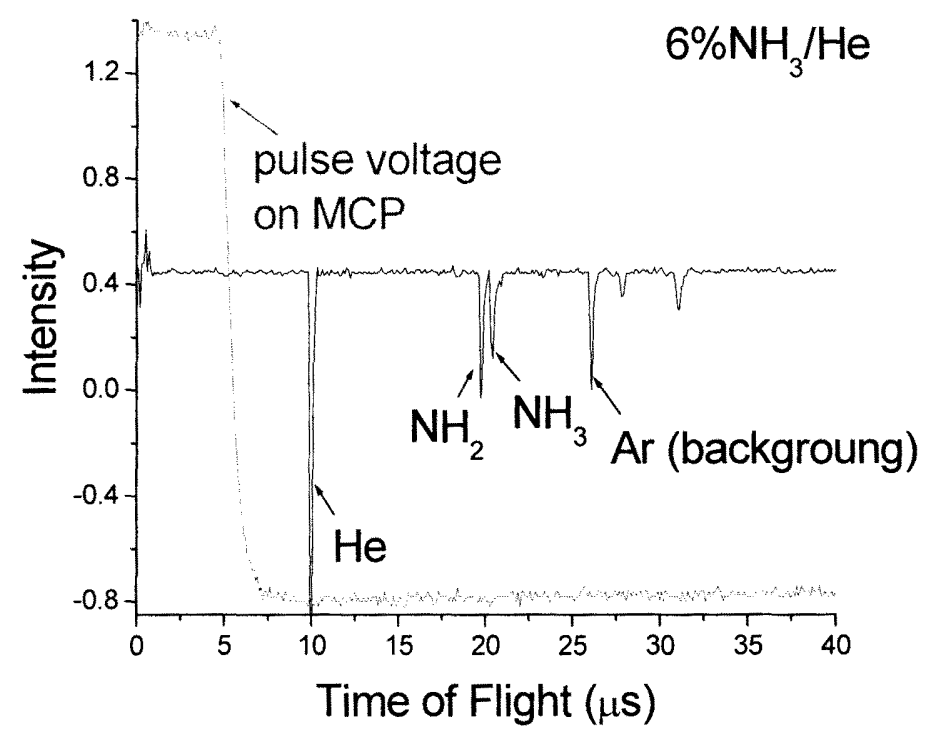

b)

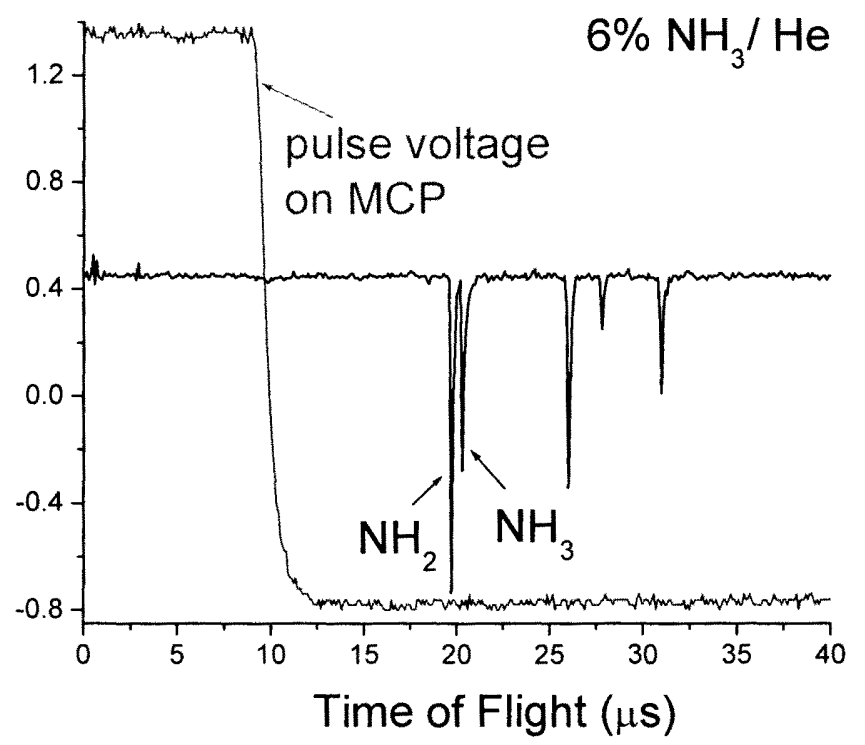

Figure 3.7: Mass spectra of $\mathrm{NH}_{3}$ obtained using He as a buffer gas. (a) Voltage on the MCP pulsed before He signal arrives at the detector (b) Voltage on the MCP pulsed after the He signal arrives, cutting the $\mathrm{He}$ signal from the spectrum. Also note the change in the $\mathrm{NH}_{2}$ and $\mathrm{NH}_{3}$ signals. The increased intensity shows that with the He signal in the spectrum the detector is saturated and produces unreliable data. 


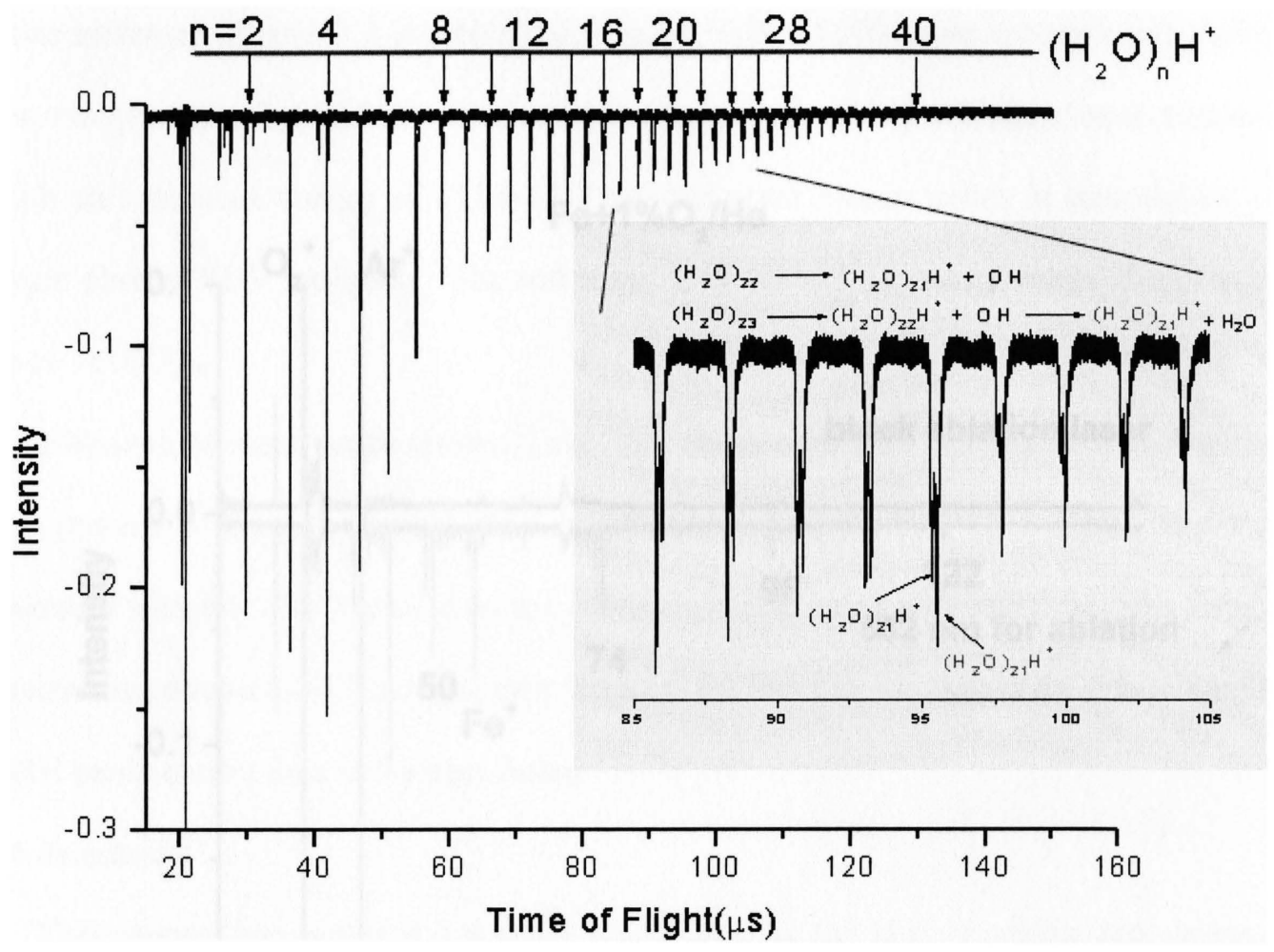

Figure 3.8: Time of flight (TOF) mass spectrum of $\left(\mathrm{H}_{2} \mathrm{O}\right)_{n}$ clusters generated by $46.9 \mathrm{~nm}$ single photon ionization with $\sim 10 \mathrm{~ns}$ spectral resolution up to $\sim\left(\mathrm{H}_{2} \mathrm{O}\right)_{40}$. 


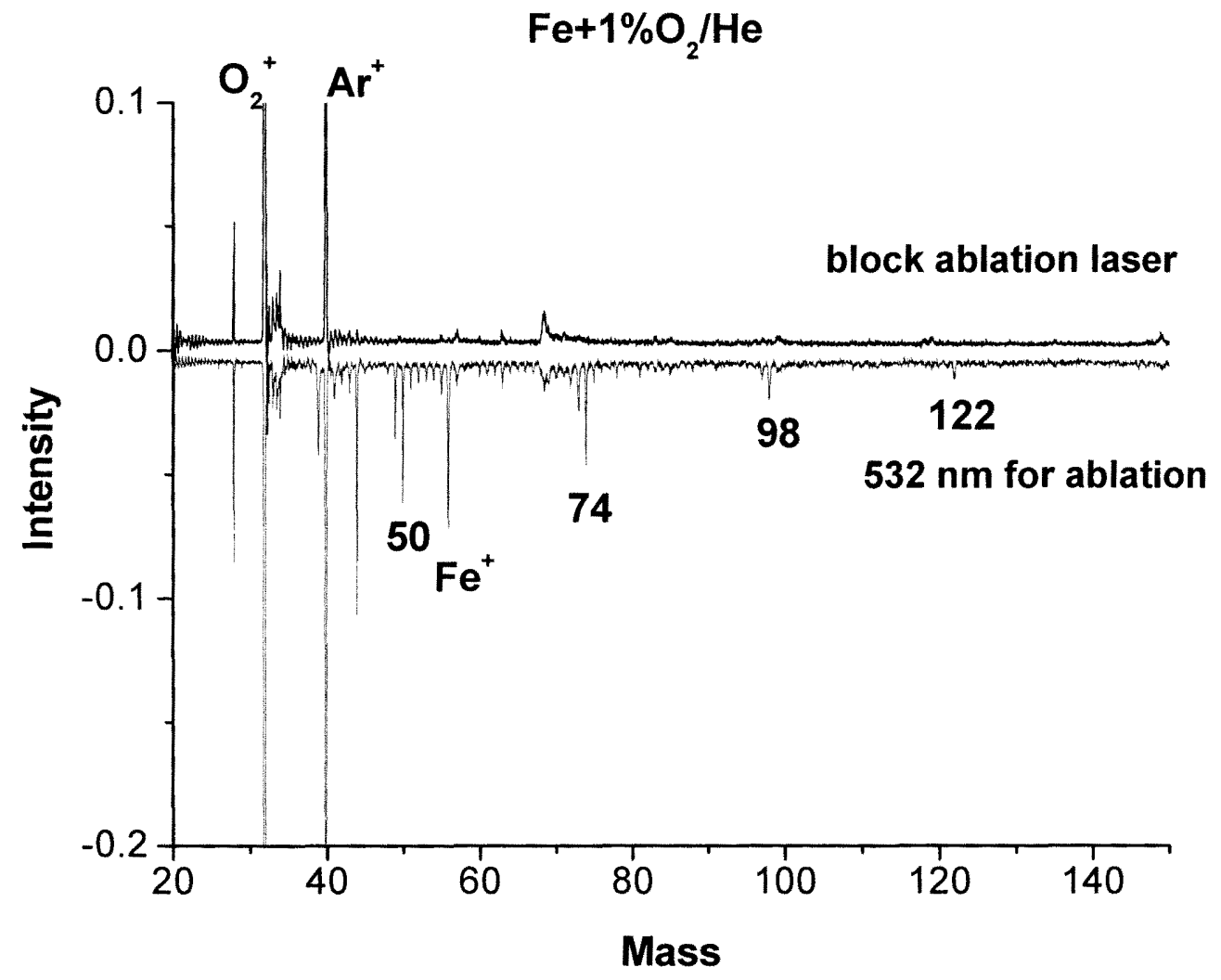

Figure 3.9: First results of a transition metal signal created by the ablation process using a Fe metal foil as a target. Spectral resolution is $\sim 10$ ns. 
ease in creating the clusters to be studied, and because they are fragile with respect to multiphoton ionization. The soft x-ray laser proved to be a worthy tool in the study of these systems. Figure 3.8 presents the time of flight (TOF) mass spectrum of $\left(\mathrm{H}_{2} \mathrm{O}\right)_{n}$ clusters generated by $46.9 \mathrm{~nm}$ single photon ionization, where $n$ is the cluster number. With an ionization energy of $\sim 11.0-11.7 \mathrm{eV}$, the water cluster series is inaccessible to single photon VUV radiation. The soft $\mathrm{x}$-ray laser provides a nicely resolved spectrum up to $\sim\left(\mathrm{H}_{2} \mathrm{O}\right)_{40}$.

Although no metal oxide clusters have been observed to date, spectra have been taken that present a signal from transition metals seen in figure 3.9. This signal shows a spectrum with iron $\left(\mathrm{Fe}^{+}\right)$created by the ablation process using a $\mathrm{Fe}$ metal foil as a target. Spectral resolution is $\sim 10 \mathrm{~ns}$. This data supports the fact that we should be able to obtain metal oxide cluster data in the near future.

\subsection{Summary}

This chapter has explored a specific application for the high repetition rate desktop soft x-ray source, namely the study of transition metal oxide clusters using TOFMS. The importance of metal oxide studies in summarized by: 1) clusters provide a theoretical and experimental bridge between the molecular and nanostructure regimes; 2) clusters provide a path to the explanation of catalytic activity under isolated, controlled, and reproducible conditions: and 3) these clusters provide a detailed mechanistic model for condensed phase and surface catalytic reactivity and reactions. Multiphoton ionization effects such as fragmentation and loss of parent cluster mass information give rise to the need for a single photon ionization source. With a photon energy of $26.5 \mathrm{eV}$, the EUV source described in this thesis is capable of producing single photon ionization of any 
metal oxide cluster, hydrogen bonded cluster, and small molecule that typically have ionization energies in the range of 7-12 eV. Several mass spectra of small molecules are obtained using the EUV laser as the ionization source demonstrating the feasibility of the use of such a source in photochemistry experiments. 


\section{References}

[3.1] M. Foltin, G. J. Stueber, and E. R. Bernstein, "On the growth dynamics of neutral vanadium oxide and titanium oxide clusters," J. Chem. Phys. 111, 9577 (1999).

[3.2] A.W. Castleman, http://research.chem.psu.edu

[3.3] A.W. Castleman, and K.H. Bowen, "Clusters: Structure, Energetics, and Dynamics of Intermediate States of Matter," J. Phys. Chem. 100, 12911 (1996).

[3.4] Y. Matsuda, and E.R. Bernstein, "On the Titanium Oxide Neutral Cluster Distribution in the Gas Phase: Detection through $118 \mathrm{~nm}$ Single-Photon and $193 \mathrm{~nm}$ Multiphoton Ionization," J. Phys. Chem. 109, 314 (2005).

[3.5] B. A. Collins, K. Athanassenas, D. M. Rayner, and P. A. Hackett, "Absorption spectra of small niobium and gold clusters measured by photodepletion of their rare gas van der Waals complexes: some preliminary experiments," Z. Phys. D 26, 36 (1993).

[3.6] L. Lian, S. A. Mitchell, P. A. Hackett, and D. M. Rayner, "Size dependence of mobility on the surface of metal clusters: Ammonia on $\mathrm{Ag}_{10}$ and $\mathrm{Ag}_{16}$," J. Chem. Phys. 104, 5338 (1996).

[3.7] E. K. Parks, B. J. Winter, T. D. Klots, and S. J. Riley, "The structure of nickel clusters," J. Chem. Phys. 94, 1882 (1991).

[3.8] D.N. Shin, Y. Matsuda, and E.R. Bernstein, "On the iron oxide neutral cluster distribution in the gas phase. II. Detection through $118 \mathrm{~nm}$ single photon ionization," J. Chem. Phys. 120, 4157 (2004).

[3.9] R.M. Jordan Website, http://www.rmjordan.com

[3.10] Yu.A. Uspenskii, V.E. Levashov, A.V. Vinogradov, A.I. Fedorenko, V.V. Kondratenko, Yu.P. Pershin, E.N. Zubarev, V.Yu. Fedotov, "High-reflectivity multilayer mirrors for a vacuum-ultraviolet intervalof 3550nm," Optics Letters 23, 771 (1998).

[3.11] Burle Website, http://www.burle.com/mcp_pmts.htm

[3.12] Stanford Website, http://www.thinksrs.com 


\section{CHAPTER 4}

\section{SUMMARY AND FUTURE WORK}

In summary, the high repetition rate $(12 \mathrm{~Hz})$ operation of a transportable desk-top size $46.9 \mathrm{~nm}$ laser that is electrically triggered for synchronization with external events has been demonstrated. To the author's knowledge this is the first demonstration of a high repetition soft $\mathrm{x}$-ray laser that can maintain an output energy of $>10 \mu \mathrm{J}$ for up to $210^{4}-3$ $10^{4}$ shots of continuous repetitive operation. This new type of portable short wavelength laser is of interest for numerous application including experiments in photochemistry, materials characterization, nano-patterning, high resolution imaging and others.

Demonstrative single-photon ionization spectroscopy experiments of nanoclusters have been conducted. These included the study of water clusters and other hydrogen bonded systems. With this $26.5 \mathrm{eV}$ photon source we were able to readily ionize water, methanol, and ammonia which require 7-12 eV of energy to ionize. Mass spectra of several other small molecules were also obtained using this ionization source. These studies also provided a good test bed for the development of a reliable soft x-ray photoionization source that can be used in many photophysics and photochemistry applications, leading the way to its widespread use as a tool in many laboratories.

A specific application for this high repetition rate desktop soft $\mathrm{x}$-ray source that we plan to explore as part of future work is the study of transition metal oxide clusters using time of flight mass spectroscopy (TOFMS). Metal-oxide clusters are of high interest as they can provide an avenue to the better understanding of catalytic processes that are used in the fabrication of many important chemicals of industrial and commercial use. The compact 
$46.9 \mathrm{~nm}$ soft x-ray source described in chapter 2 is a good contender for the single photon ionization of any metal oxide clusters, which typically have an ionization energy in the range of $6-12 \mathrm{eV}$. 